\title{
Macrophage-mediated vascular permeability via VLA4/VCAM1 pathway dictates ascites development in ovarian cancer
}

\author{
Shibo Zhang, ${ }^{1}$ Bingfan Xie, ${ }^{2}$ Lijie Wang, ${ }^{1}$ Hua Yang, ${ }^{2}$ Haopei Zhang, ${ }^{1}$ Yuming Chen, ${ }^{1}$ Feng Wang, ${ }^{2}$ Changqing Liu, ${ }^{2}$ and Huanhuan He \\ 'Cuangdong Provincial Key Laboratory of Biomedical Imaging and Guangdong Provincial Engineering Research Center of Molecular Imaging, The Fifth Affiliated Hospital, Sun Yat-sen University, Zhuhai, \\ China. 'Department of Gynaecology, The Fifth Affiliated Hospital of Sun Yat-sen University, Zhuhai, China.
}

The development of ascites correlates with advanced stage disease and poor prognosis in ovarian cancer. Vascular permeability is the key pathophysiological change involved in ascites development. Previously, we provided evidence that perivascular M2-like macrophages protect the vascular barrier through direct contact with endothelial cells (ECs). Here, we investigated the molecular mechanism and its clinical significance in the ovarian cancer setting. We found that upon direct coculture with the endothelium, M2 macrophages tuned down their VLA4 and reduced the levels of VCAM1 in ECs. On the other hand, ectopically overexpressing VLA4 in macrophages or VCAM1 in ECs induced hyperpermeability. Mechanistically, downregulation of VLA4 or VCAM1 led to reduced levels of RAC1 and ROS, which resulted in decreased phosphorylation of PYK2 (p-PYK2) and VE-cadherin (p-VE-cad), hence enhancing cell adhesion. Furthermore, targeting the VLA4/VCAM1 axis augmented vascular integrity and abrogated ascites formation in vivo. Finally, VLA4 expression on the macrophages isolated from ascites dictated permeability ex vivo. Importantly, VLA4 antibody acted synergistically with bevacizumab to further enhance the vascular barrier. Taking these data together, we reveal here that M2 macrophages regulate the vascular barrier though the VCAM1/RAC1/ROS/p-PYK2/p-VE-cad cascade, which provides specific therapeutic targets for the treatment of malignant ascites.

\section{Introduction}

Ovarian cancer (OC) is the fifth leading cause of cancer-related death, and it has the highest mortality rate among all gynecological cancers worldwide (1). Ascites, fluid accumulation in the peritoneal cavity, has been observed in $44.1 \%$ of patients at the time of epithelial OC (EOC) diagnosis $(2,3)$, and it develops more frequently in EOC than in any other tumor types (4). Ascites can cause deterioration in the quality of life of patients, causing abdominal pain and respiratory distress $(5,6)$. The onset and progression of ascites correlate with poor prognosis and disease recurrence (7). It is believed that ascites formation is due to increasing i.p. vascular permeability combined with impaired lymphatic drainage $(6,8)$. However, current management of malignant ascites based on these understandings is unsatisfactory, making it imperative to gain more insight into the mechanism of ascites development so that more effective treatment strategies can be developed.

Changes in vascular permeability play an important role in tissue fluid homeostasis (9). In tumor tissues, tumor vessels are often intricate, barely covered by mural cells, and loosely associated with

Authorship note: SZ, BX, LW, and HY contributed equally to this work as co-first authors. $\mathrm{CL}$ and $\mathrm{HH}$ contributed equally to this work as co-senior authors.

Conflict of interest: The authors have declared that no conflict of interest exists. Copyright: () 2021, American Society for Clinical Investigation.

Submitted: May 18, 2020; Accepted: November 25, 2020; Published: February 1, 2021

Reference information: J Clin Invest. 2021;131(3):e140315.

https://doi.org/10.1172/JCl140315. the basement membrane. Therefore, increased capillary permeability, which is orchestrated by ample vascular endothelial growth factor (VEGF) secreted into the microenvironment, leads to the overflow of ascites $(10,11)$. However, our previous study suggests a strategy for regulating vascular permeability, i.e., M2-like macrophages can protect the vascular barrier through direct contact with the endothelium (12). This observation raises the question of whether macrophage-mediated vascular permeability also participates in pathological events, such as ascites development in OC.

Integrins are a large group of heterodimeric adhesion molecules that are indispensable regulators of the vascular barrier (13-15). Integrin $A_{4} \beta_{1}$, also known as very late antigen-4 (VLA4), is primarily expressed on leukocytes and often binds to vascular cell adhesion molecule 1 (VCAM1) on endothelial cells (ECs) to mediate leukocyte trafficking upon inflammation. The activation of VCAM1 promotes the phosphorylation of VE-cadherin (pVE-cad), resulting in the loss of cell adhesion (16). Utilizing this signaling pathway from the opposite perspective, we demonstrate here that M2 macrophages inhibited endothelial VCAM1 expression upon direct contact, which suppressed the downstream cascade and dampened $\mathrm{p}-\mathrm{VE}$-cad, thus protecting the vascular barrier. Targeting the VLA4/VCAM1 axis could attenuate vascular permeability both in vitro and in vivo and, more importantly, could inhibit ascites formation in an OC animal model. Our findings have uncovered the molecular mechanism behind M2 macrophage-mediated vascular permeability and provided new strategies for treating ascites in OC. 
A

Cytokine-cytokine receptor interaction Chemokine signaling pathway TNFsignaling pathway

Toll-like receptor signaling pathway IL-17 signaling pathway

Hematopoietic cell lineage

NF-kappa B signaling pathway

Transcriptional misregulation in cancer

NOD-like receptor signaling pathway

Cell adherin molecules (CAMs)

Cytosolic DNA-sensing pathway African trypannosomiasis Influenza A

C
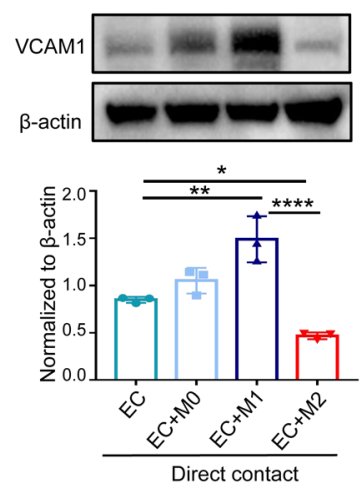

E
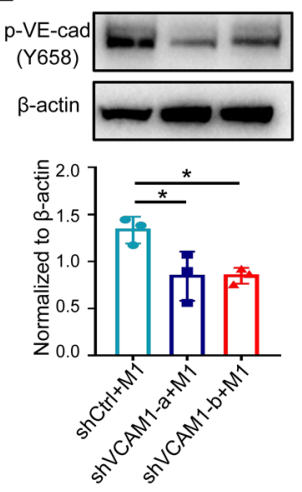

I

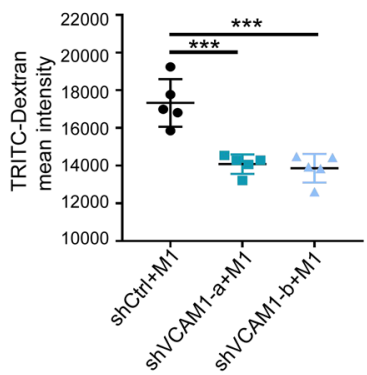

J

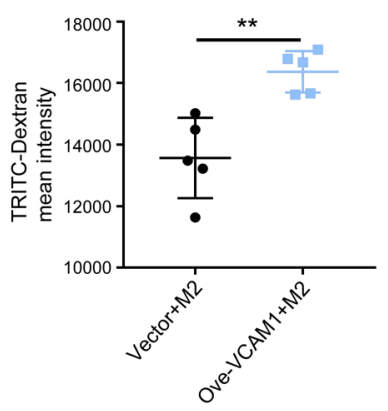

EC (M1) VS EC (M2)

Qvalue

0.00000
-0.00002

$-0.00004$

$-0.00006$

$-0.00008$

$-0.00010$

$-0.00012$

Gene Number

- 9

21

32

44

55
B

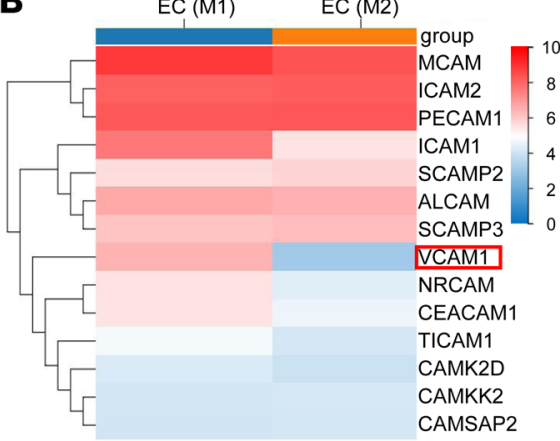

D

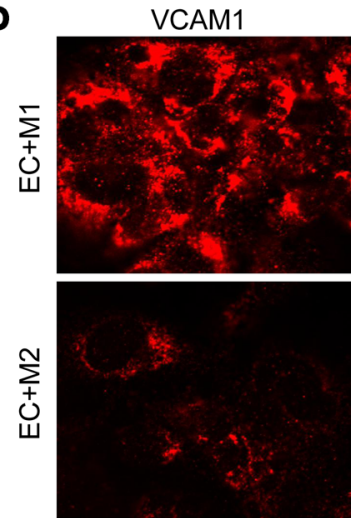

$\mathbf{F}$

p-VE-cad (Y658)
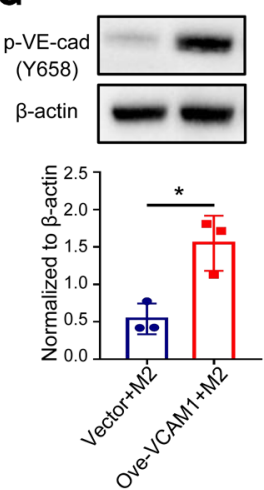

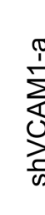
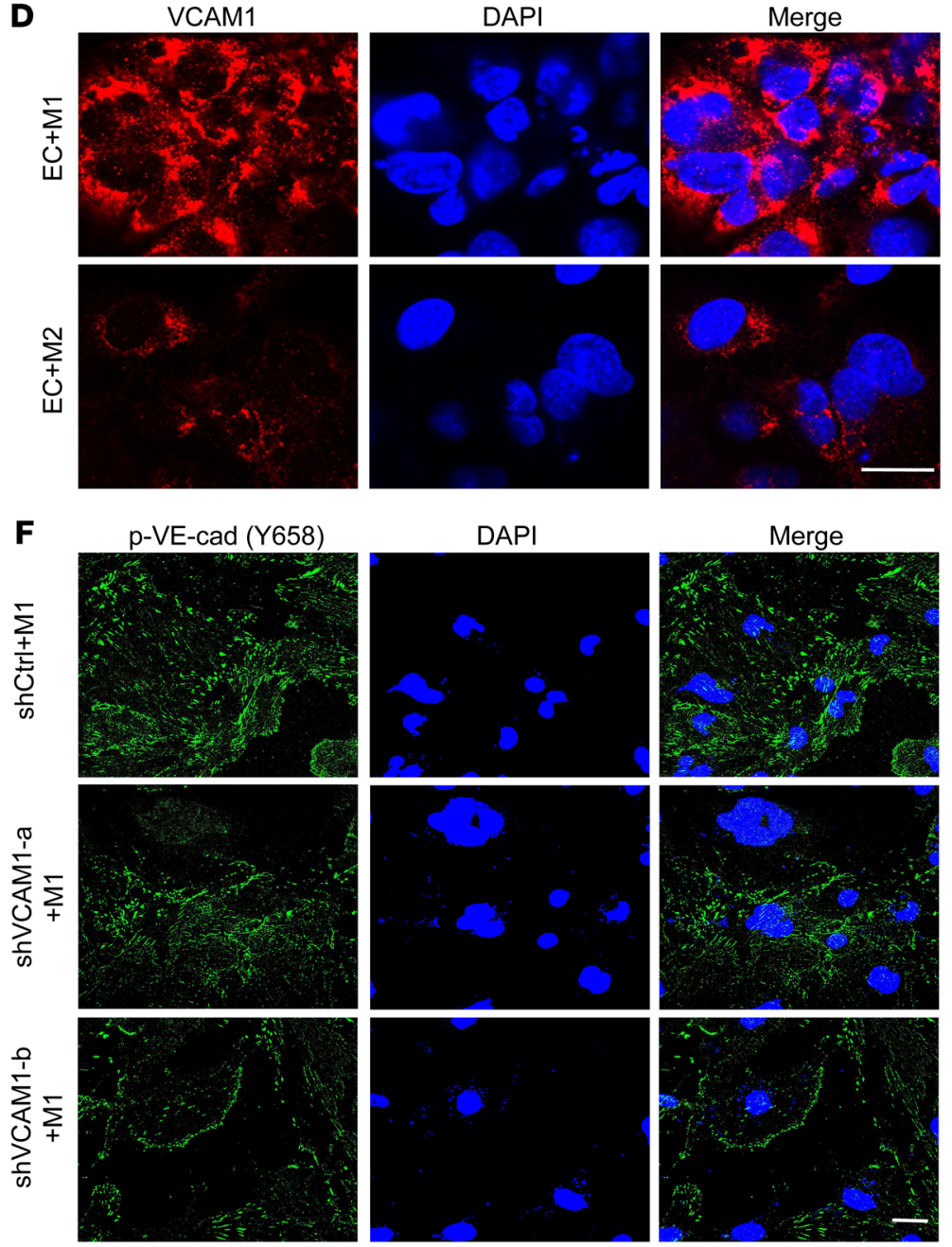

H p-VE-cad (Y658)
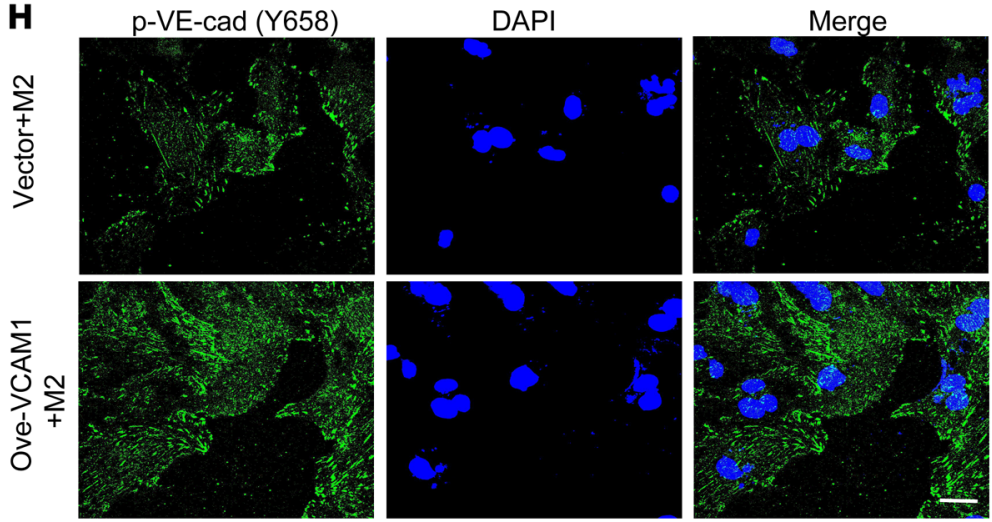
Figure 1. M2 macrophages induce hypopermeability by downregulating VCAM1 in ECs (A) KEGG pathway analysis of DEGs in M1 macrophagetreated HUVECs versus M2 macrophage-treated HUVECs. (B) Gene expression heatmap of differentially expressed CAM genes from $\mathbf{A}$. (C) Expression of VCAM1 in HUVECs detected by Western blot in different cocultures ( $n=$ 3). (D) Localization of VCAM1 protein (red) by immunofluorescence analysis. DAPI stains cell nucleus. Scale bar: $20 \mu \mathrm{m}$. (E) p-VE-cad expression in M1 macrophage-cocultured HUVECs that were transiently transfected with VCAM1-specific shRNAs (shVCAM1-a, shVCAM1-b) or a control shRNA (shCtrl) $(n=3)$. (F) Immunofluorescence analysis of p-VE-cad in M1 macrophage-cocultured HUVECs with VCAM1 knocked down. Scale bar: $20 \mu \mathrm{m}$. (G) p-VE-cad expression in M2 macrophage-cocultured HUVECs that were transiently transfected with a VCAM1-specific vector (Ove-VCAM1) or a control vector $(n=3)$. (H) Immunofluorescence analysis of $p-V E-c a d$ in M2 macrophage-cocultured HUVECs with VCAM1 overexpressed. Scale bar: 20 $\mu \mathrm{m}$. (I) TRITC-Dextran tracer fluorescence from M1 macrophage-cocultured HUVECs that were transfected with VCAM1 knockdown shRNAs (shVCAM1-a, shVCAM1-b) or a control shRNA (shCtrl; $n=5$ ). (J) TRITC-dextran tracer fluorescence from M2 macrophage-cocultured HUVECs that were transfected with VCAM1-specific vector (Ove-VCAM1) or control vector ( $n=$ 5). Data represent 3 independent experiments. Results are shown as mean \pm SD. ${ }^{*} P<0.05 ;{ }^{* *} P<0.01$; ${ }^{* *} P<0.001 ;{ }^{* * *} P<0.0001$, 1-way ANOVA (C, E, I) and Student's $t$ test (G and J).

\section{Results}

M2 macrophages induce hypopermeability by downregulating VCAM1 in ECs. Our previous study showed that perivascular M2-like macrophages could protect against vascular permeability, but M1s could not. To explore the mechanism underlying the effect of macrophages on vascular permeability, we first evaluated the proteins involved in the regulation of vascular integrity, such as the adherens junction protein VE-cadherin and the tight junctional protein $\mathrm{ZO}-1$. We found that the human umbilical vein EC (HUVEC) layer cocultured with M2 macrophages (THP-1) for 24 hours experienced a significant reduction in the levels of $\mathrm{p}-\mathrm{VE}$-cad at the Tyr658 site (Y658; Supplemental Figure 1A; supplemental material available online with this article; https://doi.org/10.1172/ JCI140315DS1). However, the expression of ZO-1 (Supplemental Figure 1B) and the level of p-VE-cad at the Tyr731 site (Y731) were unchanged (Supplemental Figure 1C).

We then compared the gene expression profile of HUVECs that had been cocultured with different subtypes of macrophages. Among the profiles of differentially expressed genes (DEGs), we focused on cell adhesion molecules (CAMs), since macrophage-mediated permeability requires direct cell-cell contact (Figure 1A). Among all the CAMs, VCAM1 was the most markedly changed (Figure 1B). To validate this result, we isolated ECs from the coculture system to detect the expression of VCAM1 mRNA and protein and obtained consistent conclusions (Supplemental Figure 1, D and E). Notably, expression of VCAM1 protein in HUVECs was downregulated only upon direct contact with M2 THP-1 macrophages, as detected by both Western blot and immunofluorescence staining (Figure 1, C and D). This finding was validated by coculturing murine macrophages (RAW264.7) with a murine EC line (C166; Supplemental Figure 1, F and G). Interestingly, such an effect vanished in the coculture performed via a Transwell system (Supplemental Figure 1H), suggesting contact-dependent regulation of endothelial VCAM1 by M2 macrophages. We also measured the levels of soluble VCAM1 (sVCAM1), and found no difference in HUVECs cocultured with M1 versus M2 macrophages (Supplemental Figure 1I). To validate the crucial role of VCAM1 in regulating macrophage-mediated permeability, we knocked down VCAM1 expression in HUVECs using shRNAs (Supplemental Figure 1J) and observed attenuated p-VE-cad in EC-M1 macrophage coculture (Figure 1E). Immunofluorescence staining confirmed the downregulation of p-VE-cad upon VCAM1 knockdown (Figure 1F). Alternatively, when applying a VCAM1 inhibitor K-7174 to block VCAM1 expression in coculture, the $\mathrm{p}$-VE-cad level was clearly abrogated (Supplemental Figure 1K). Conversely, VCAM1 overexpression in HUVECs and murine ECs promoted $\mathrm{p}$-VE-cad (Figure 1, G and H, and Supplemental Figure 1, L and M).

Next, we sought to confirm that VCAM1 could functionally affect macrophage-mediated permeability. Macrophages and ECs were cocultured in the upper well of a Transwell, and dextran labeled with TRITC fluorescent dye was allowed to pass through the barrier, which was then collected in the lower chamber and quantified (17). The data revealed that there was much less dextran that passed through in the VCAM1 knockdown group relative to that of the control group (Figure 1I), whereas increased flux of dextran was observed in the VCAM1-overexpressing coculture (Figure 1J). Together, these findings demonstrate that macrophages exert their functions of regulating the vascular barrier by affecting VCAM1 expression on the endothelium.

VLA4 expression and activation are dampened in M2 macrophages cocultured with ECs. To determine which molecule in M2 macrophages dictates differential regulation in ECs, we scrutinized the gene expression profiles of both subtypes of macrophages isolated from coculture and found that the pathway associated with leukocyte transendothelial migration was enriched and downregulated in M2 macrophages (Figure 2A). Among all the genes participating in leukocyte transendothelial migration, it was noteworthy that the expression of VLA4, the ligand of VCAM1, decreased markedly in M2 macrophages (Figure 2B). We confirmed this result by Western blot, immunofluorescence staining, and quantitative reverse-transcription PCR (qRT-PCR) (Figure 2, C and D, and Supplemental Figure 2, A and B). VLA4 protein expression was also reduced in M2-polarized murine macrophages cocultured with murine endothelium (Supplemental Figure 2, C and D). It should be noted that the expression of the active form of VLA4 in cocultured M2 macrophages was also lessened compared with that in the cocultured M1 macrophage (Figure 2E).

To validate the regulatory effects of macrophage VLA4 on EC barriers, THP-1 or RAW264.7 cells were subjected to inhibited or excessive VLA4 expression by shRNAs (Supplemental Figure 2, E and F) or overexpression plasmids (Supplemental Figure 2, G and $\mathrm{H})$, followed by coculturing with ECs. Consistent with the VCAM1 data, silencing VLA4 in macrophages or pretreatment of macrophages with the VLA4 inhibitor, CDP323, attenuated p-VE-cad upon coculture in both HUVECs (Figure 2, F and G) and C166s (Supplemental Figure 2, I and J); however, increased p-VE-cad was detected when VLA4 was overexpressed or VLA4 agonist, THIO019, was administered (Figure 2, H-J, and Supplemental Figure 2, K and L). Dextran permeability assay showed that suppressing VLA4 expression in macrophages either by treating with its inhibitor (CDP323) or antibody (PS/2) blocked the dextran from crossing the EC barrier, while enhancing VLA4 expression or 
A

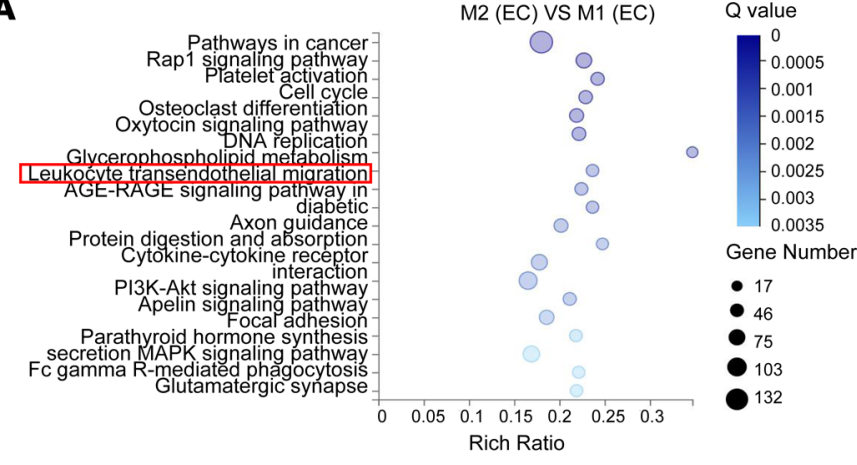

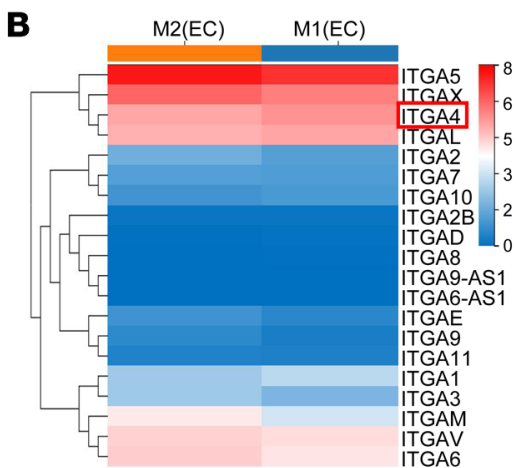
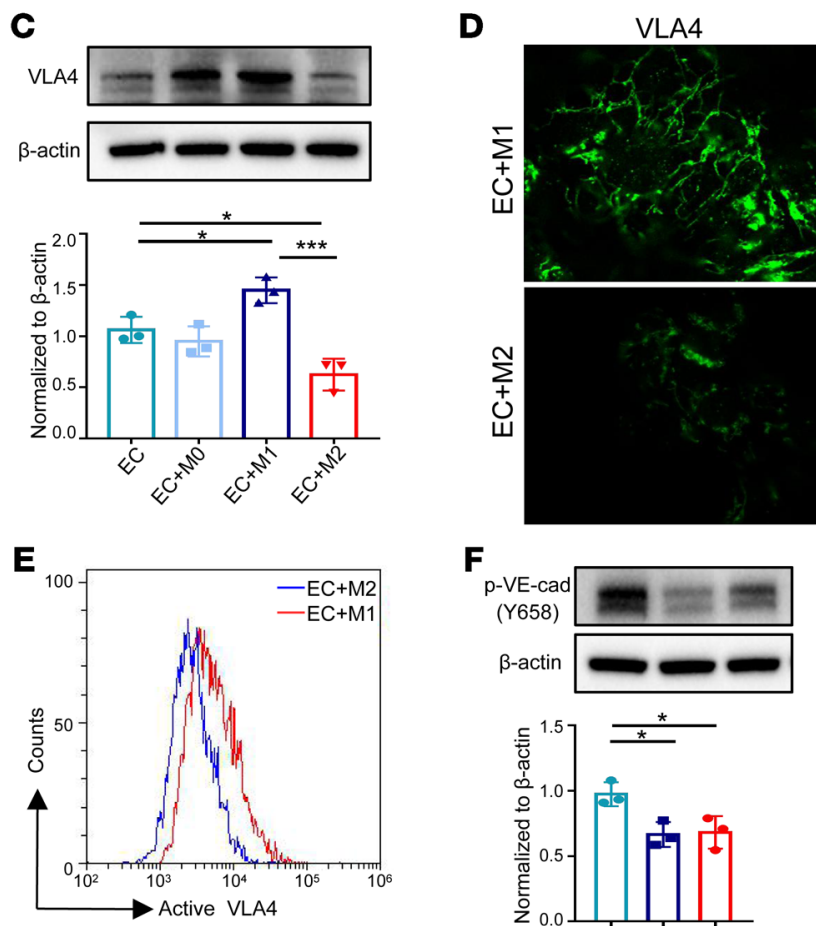

$\mathbf{F}$
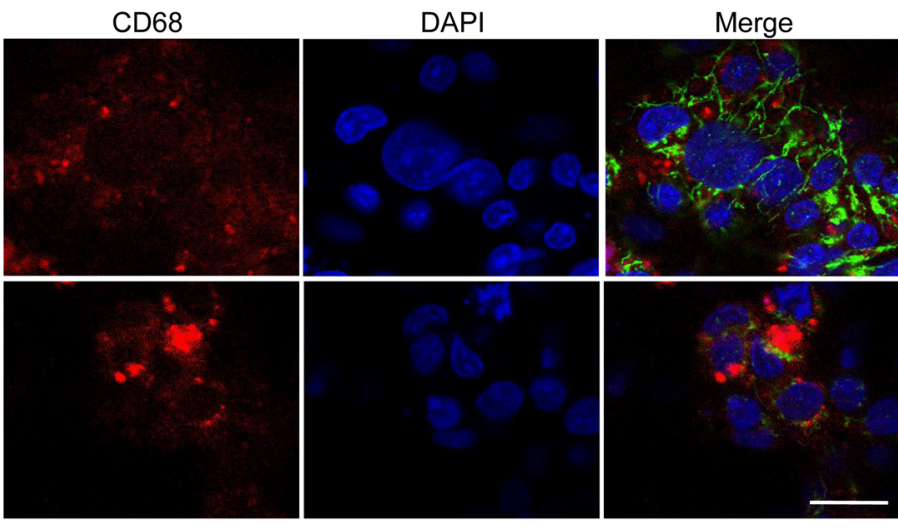

H
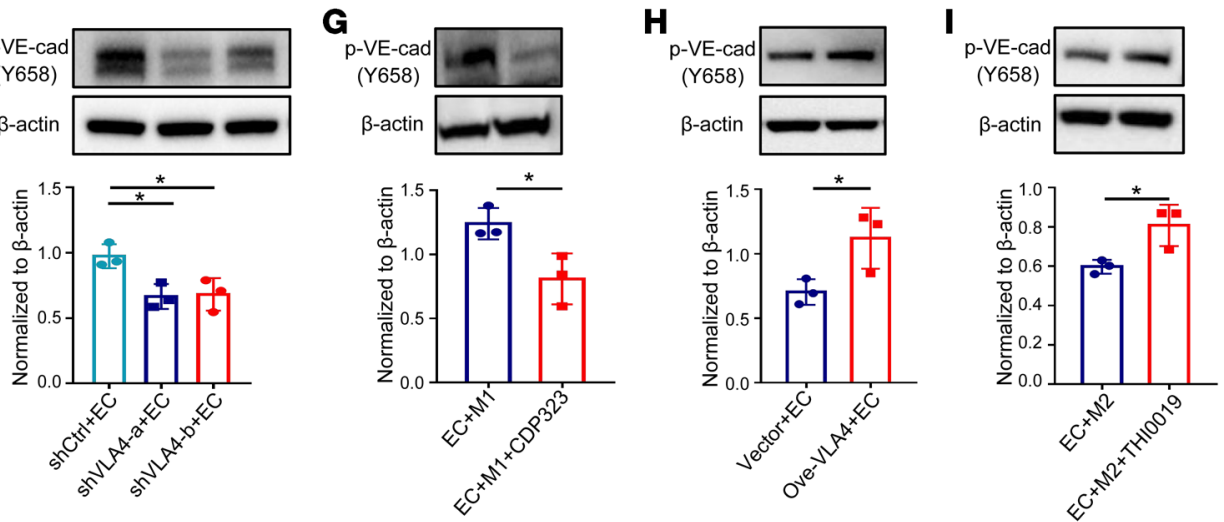

DAPI

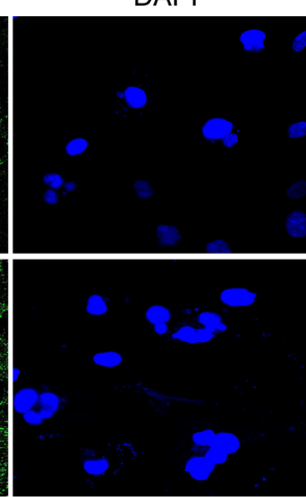

Merge

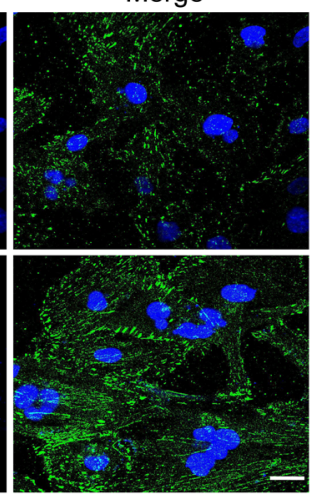

$\mathbf{K}$

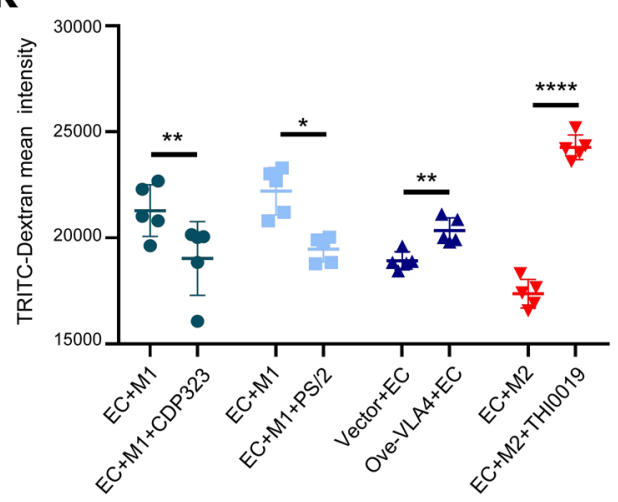


Figure 2. Decreased VLA4 activation in M2 macrophages cocultured with ECs. (A) KEGG pathway analysis of DEGs when comparing M2 versus M1 macrophages from cocultures. (B) Gene expression heatmap of differentially expressed integrin genes from A. (C) Expression of VLA4 in THP-1 macrophages detected by Western blot in different cocultures $(n=3)$. (D) Localization of VLA4 protein (green) in THP-1 macrophages by immunofluorescence analysis. CD68 (red) stains macrophages. DAPI stains cell nucleus. Scale bar: $20 \mu \mathrm{m}$. (E) Representative graph of flow cytometric analysis of active VLA4 levels in different subtypes of THP-1 macrophages from cocultures. (F) p-VE-cad expression in HUVECs cultured with macrophages that were transiently transfected with VLA4-specific shRNAs (shVLA4-a, shVLA4-b) or a control shRNA (shCtrl) $(n=3)$. (C) p-VE-cad expression in HUVECs from M1 macrophage-coculture system treated with $\operatorname{CDP323}(n=3)$. (H) p-VE-cad expression in HUVECs cocultured with macrophages that were transiently transfected with a VLA4-specific vector (Ove-VLA4) or a control vector $(n=3)$. (I) p-VE-cad expression in HUVECs from M2 macrophage-coculture system treated with THI0019 ( $n=3)$. (J) Immunofluorescence analysis of p-VE-cad expression in HUVECs cultured with macrophages that were transiently transfected with a VLA4-specific vector (Ove-VLA4) or a control vector. Scale bar: $20 \mu \mathrm{m}$. (K) TRITC-dextran tracer fluorescence from coculture systems in which macrophages transiently transfected with a VLA4-specific vector (OveVLA4) or pretreated with THI0019, PS/2, and CDP323 are compared with the respective control $(n=5)$. Data represent 3 independent experiments. Results are shown as mean $\pm \mathrm{SD}$. ${ }^{*} P<0.05 ;{ }^{* *} P<0.01$; ${ }^{* *} P<0.001$; ${ }^{* * *} P<0.0001$, Student's $t$ test (G-I and $\mathbf{K}$ ) and 1-way ANOVA (C and $\mathbf{F}$ ).

applying its agonist (THIO019) exacerbated EC hyperpermeability (Figure 2K). Overall, these results indicate that the level of VLA4 on macrophages contributes to the regulation of the vascular barrier.

ROS functions downstream of VCAM1 to regulate macrophage-mediated permeability. It has been reported that the accumulation of ROS could ultimately lead to endothelial dysfunction (18). To determine whether ROS could play a role in macrophage-mediated permeability, we first measured ROS expression in the coculture system. As shown in Figure 3A, endothelial ROS was largely abolished when coculturing with M2 macrophages compared with that with M1s, and this difference was further enhanced following treatment with the vascular permeator VEGF (Figure 3B). Moreover, the addition of CDP323 (Figure 3C) or K-7174 (Figure 3D) effectively blocked intracellular ROS production in HUVECs. In contrast, the ROS level in the ECs from VLA4-overexpressing coculture was higher than that in the control (Supplemental Figure 3 , A and B), suggesting that ROS was the downstream effector of M2 macrophage-mediated vascular permeability.

To confirm that the regulation of $\mathrm{p}-\mathrm{VE}-\mathrm{cad}$ by macrophages depends on ROS, ROS inhibitor $\mathrm{N}$-acetyl-L-cysteine (NAC) was added to the coculture system. To support our hypothesis, $\mathrm{p}^{-}$ VE-cad was significantly abolished in ECs cocultured with macrophages in the presence of NAC (Figure 3E and Supplemental Figure 3C), whereas p-VE-cad had an evident increase after $\mathrm{H}_{2} \mathrm{O}_{2}$ was introduced in the EC-macrophage cocultures (Figure 3F and Supplemental Figure 3D). We confirmed the above findings by immunofluorescence staining (Figure 3, G and H, and Supplemental Figure 3E). Barrier function detected by cellZscope confirmed that vascular permeability was abolished upon NAC treatment, while the barrier was markedly compromised with $\mathrm{H}_{2} \mathrm{O}_{2}$ treatment in ECs cocultured with macrophages (Figure 3, I and J, and Supplemental Figure 3F). Collectively, these data demonstrate that the VLA4/VCAM1-initiated cascade could induce vascular hyperpermeability via enhancing ROS.
Macrophage-mediated permeability is dependent on RAC1 and phosphorylated PYK2. RAS-related C3 botulinum substrate 1 (RAC1) and proline-rich tyrosine kinase 2 (PYK2) have been identified as potential regulators of $\mathrm{p}-\mathrm{VE}-\mathrm{cad}$, and they can potentially regulate ROS levels up- and downstream, respectively (19). Downregulated total RAC1 and phosphorylation of PYK2 (p-PYK2; Tyr402), but not total PYK2, were observed in the coculture system with M2 macrophages compared with that in the M1 cocultures (Figure 4A and Supplemental Figure 4, A and B). Consistent with previous results, such change was not observed in the coculture without direct contact (Supplemental Figure 4C). Immunofluorescence staining confirmed the above changes (Figure 4B). Strikingly, using VLA4 inhibitor CDP323 to treat macrophages diminished both RAC1 and p-PYK2 levels in EC-macrophage cocultures (Figure 4C and Supplemental Figure 4D). RAC1 expression levels in the coculture were increased when treating macrophages with the VLA4 agonist THIO019 (Supplemental Figure 4, E and F). Furthermore, knockdown of VCAM1 dampened RAC1 expression, while VCAM1 overexpression promoted RAC1 expression (Supplemental Figure 4, G-I). Concomitantly, reducing VCAM1 levels by treating with its inhibitor abrogated both RAC1 and p-PYK2 levels in EC-macrophage cocultures (Figure $4 \mathrm{D}$ and Supplemental Figure 4J). To prove that ROS is regulated by RAC1, we applied the RAC1 inhibitor NSC23766 and detected lower ROS level in the EC cocultures (Figure 4E and Supplemental Figure $4 \mathrm{~K}$ ). Since PYK2 is activated through ROS in ECs (20), it was expected that NAC treatment reduced p-PYK2 expression in HUVECs (Figure $4 \mathrm{~F}$ ), whereas $\mathrm{H}_{2} \mathrm{O}_{2}$ treatment increased p-PYK2 expression (Figure 4G and Supplemental Figure 4, L and M). Taken together, the above results suggest that RAC1/ROS/p-PYK2 act downstream of VCAM1 and participate in the regulation of macrophage-mediated vascular permeability.

Blocking VLA4 reduces permeability and lessens ascites in vivo. To further investigate whether VLA4 is responsible for regulating macrophage-mediated vascular permeability in vivo, we resorted to a rescue approach. First, macrophages were largely depleted using clodronate liposomes via i.p. injection in mice, and the depletion of peritoneal macrophages was verified by flow cytometry (Supplemental Figure 5A). Then, RAW264.7 cells with either suppressed or promoted levels of VLA4 were reintroduced via i.p. injection. After 2 days of reconstruction, peritoneal vascular permeability was measured by fluorescent dye that was injected intravenously and then leaked into the peritoneal cavity (Figure $5 \mathrm{~A})$. In line with the in vitro data, the leaky vascular barrier was reconstructed when VLA4 was either knocked down (Figure 5B) or blocked by a functional VLA4-blocking antibody, PS/2 (Supplemental Figure 5B). Furthermore, the leakage was more intense in the VLA4-overexpressing group compared with the control group (Figure 5C). These results proved that the VLA4 level was positively associated with vascular permeability in vivo, underscoring the therapeutic potential of targeting VLA4. Moreover, upon macrophage removal, the effect of PS/2 on vascular permeability was weakened (Figure 5D), which suggested that the effect of VLA4 on permeability was macrophage dependent.

To test the efficacy of blocking VLA4 for the treatment of ascites, we resorted to a murine OC model, HM-1. PS $/ 2(3 \mathrm{mg} / \mathrm{kg})$ was administered to the mouse abdomen according to the schematic 
A

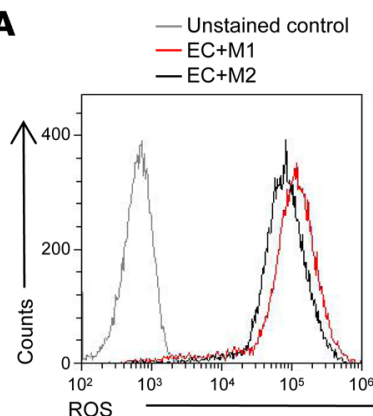

B - Unstained control - EC+M1+VEGF

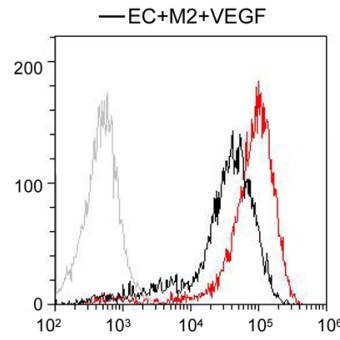

C - Unstained control - $\mathrm{EC}+\mathrm{M} 1$

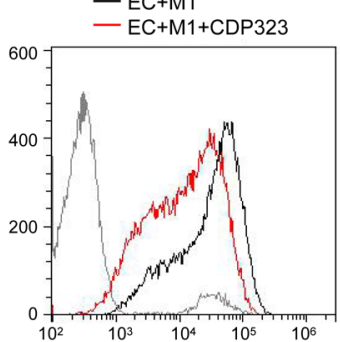

D - Unstained control

- $\mathrm{EC}+\mathrm{M} 1$ - EC+M1+K-7174

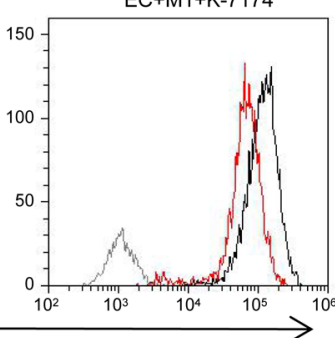

G $\quad p-V E-c a d(Y 658)$
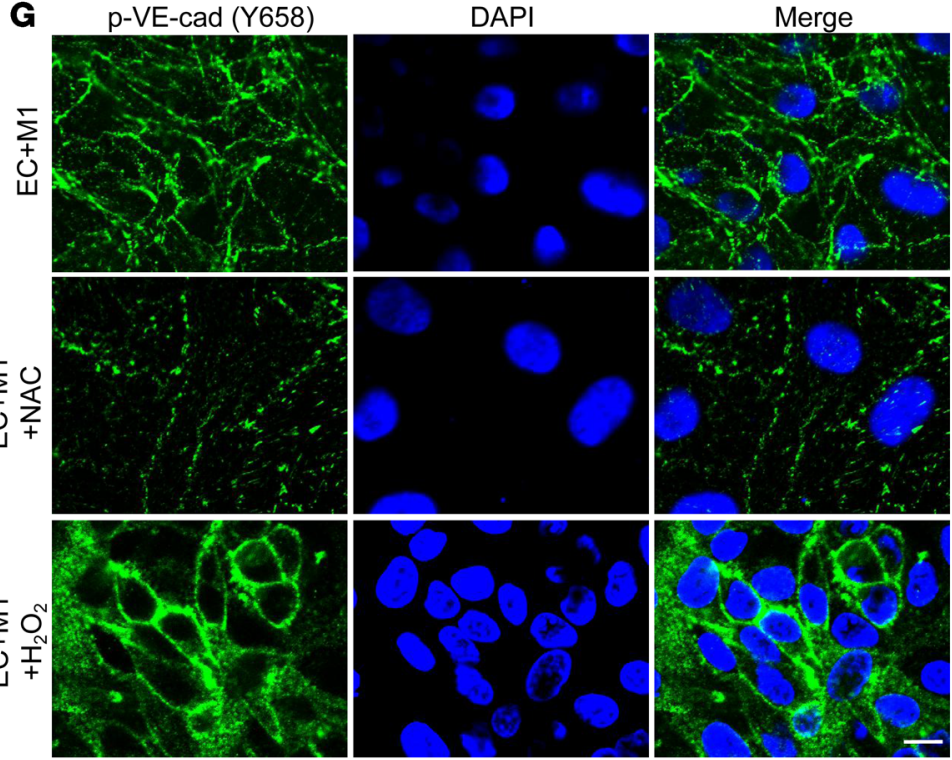

E

$\mathrm{p}-\mathrm{VE}-\mathrm{cad}$
(Y658)

$\beta$-actin
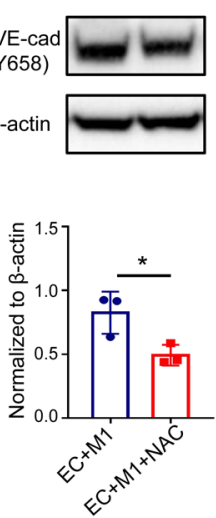

$\mathbf{F}$
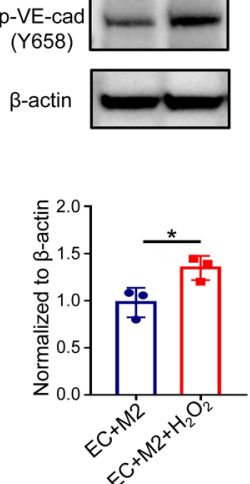
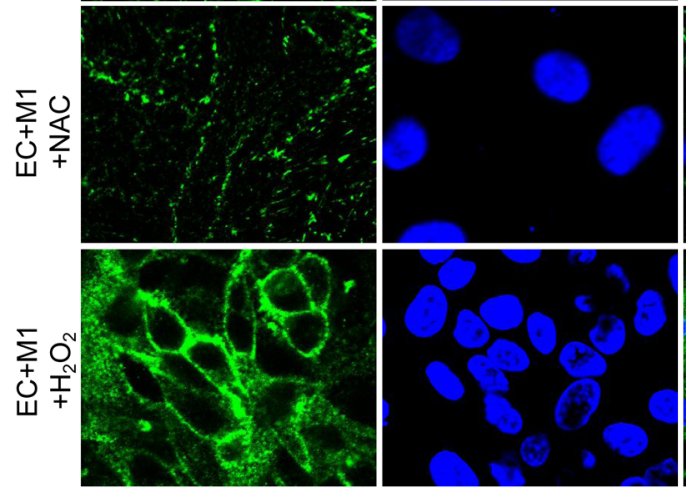

H
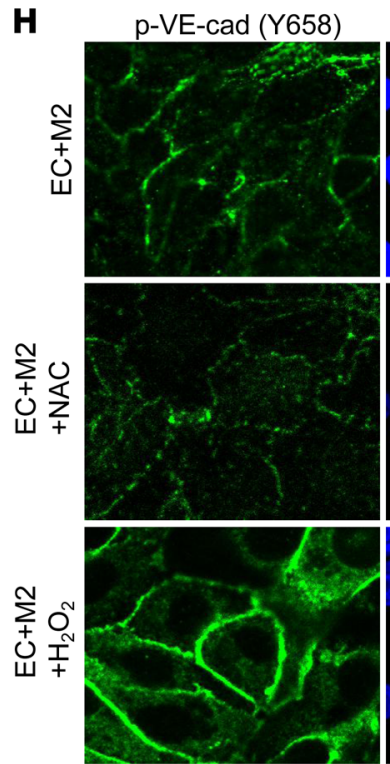

DAPI
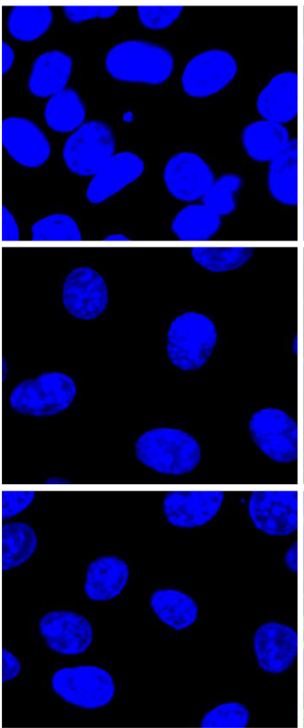

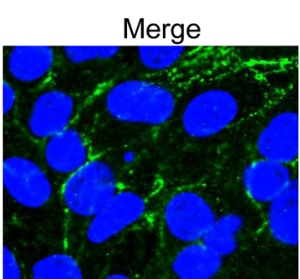

I
I -.-- $\mathrm{EC}+\mathrm{M} 1$

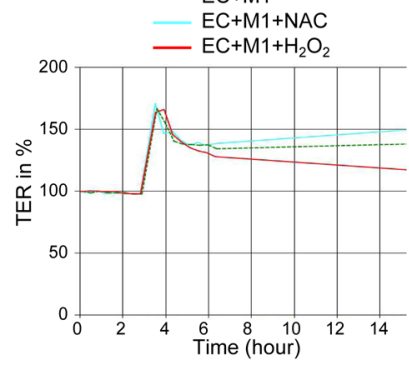

J

---- EC+M2

--- $\mathrm{EC}+\mathrm{M} 2+\mathrm{NAC}$
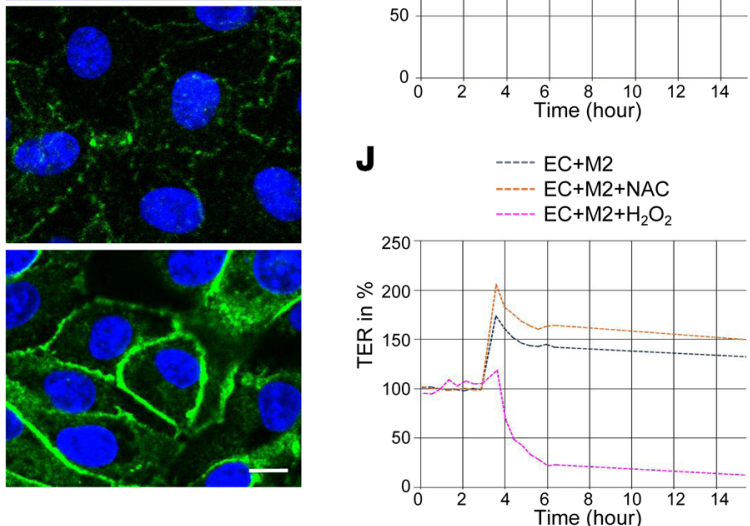

Figure 3. ROS functions downstream of VCAM1 in regulating macrophage-mediated permeability. (A-D) Representative graphs of flow cytometric analysis of ROS levels in HUVECs cocultured with different subtypes of macrophages (A), treated with VEGF (B), and in HUVECs from M1 macrophage-coculture system treated with CDP323 (C) or K-7174 (D). (E) Immunoblot analysis of p-VE-cad expression in HUVECs from M1 macrophage-cocultured system treated with NAC $(n=3)$. (F) p-VE-cad expression in HUVECs from M2 macrophage-cocultured system treated with $\mathrm{H}_{2} \mathrm{O}_{2}(n=3)$. (G and $\left.\mathbf{H}\right)$ Immunofluorescence of $\mathrm{p}-\mathrm{VE}$-cad expression in HUVECs from M1 (G) or M2 (H) macrophage-cocultured system treated with $\mathrm{NAC}$ and $\mathrm{H}_{2} \mathrm{O}_{2}$. Scale bars: $20 \mathrm{Hm}$. (I and J) Effects of $\mathrm{NAC}$ or $\mathrm{H}_{2} \mathrm{O}_{2}$ treatment in HUVECs from M1 macrophage-cocultured system (I) or M2 macrophage-cocultured system (J) measured in real time using the automated system (cellZscope). Data represent 3 independent experiments. Results are shown as mean \pm SD. ${ }^{*} P<0.05$, Student's $t$ test (E, F). 
A
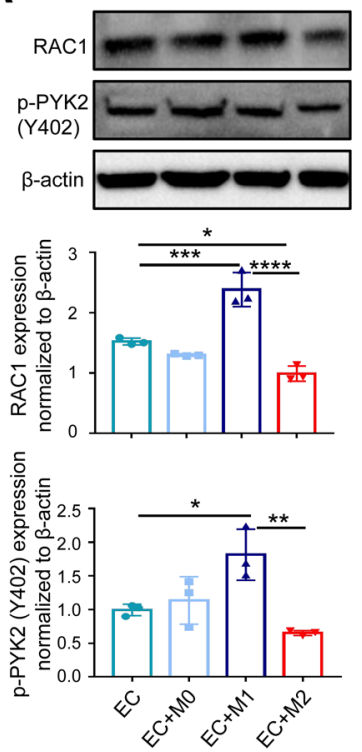

C

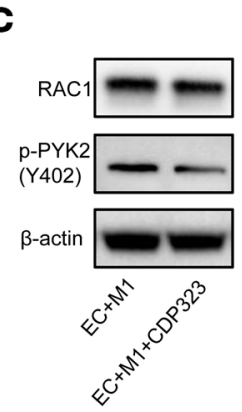

$\mathbf{F}$

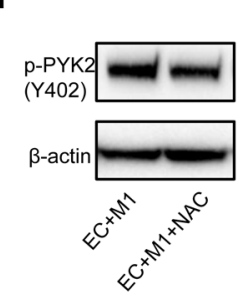

B
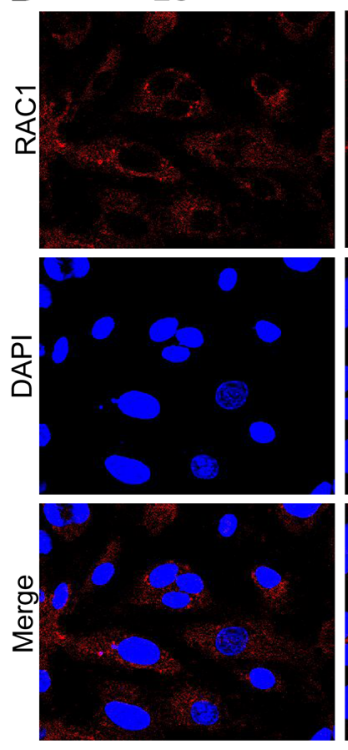

D
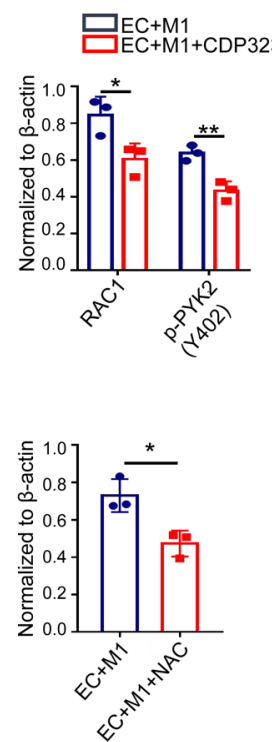

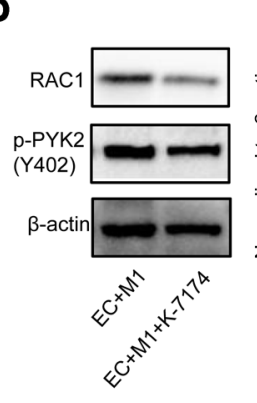

G

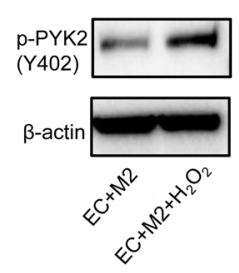

$E C+M O$
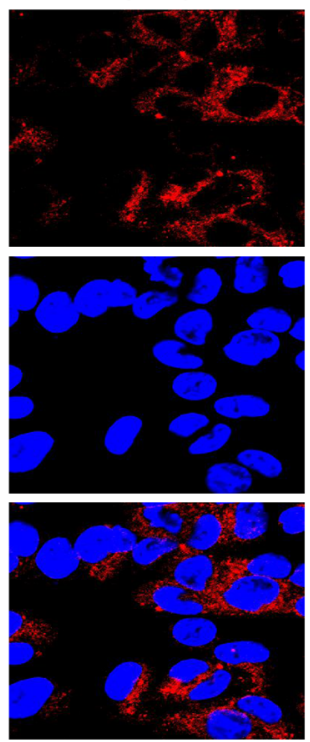

$\mathrm{EC}+\mathrm{M} 1$
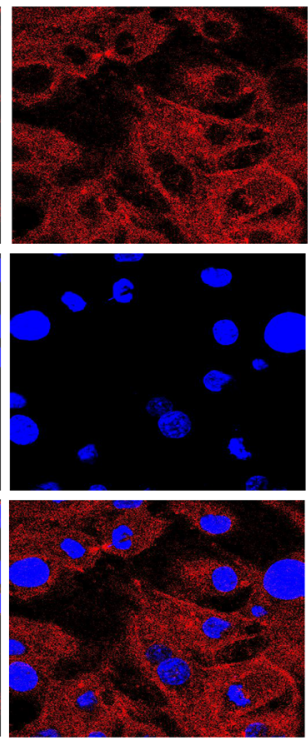

$\mathrm{EC}+\mathrm{M} 2$
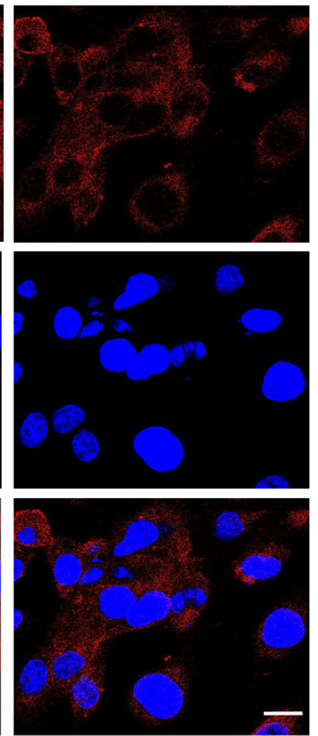

E
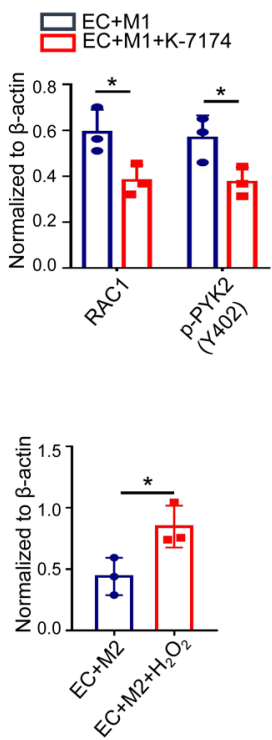

Figure 4. Macrophage-mediated permeability is dependent on RAC1 and p-PYK2. (A) Protein levels of RAC1 and p-PYK2 in HUVECs from different cocultures examined by Western blot $(n=3)$. (B) Immunofluorescence analysis of RAC1 in HUVECs and HUVECs cultured with different subtypes of macrophages. Scale bar: $20 \mu \mathrm{m}$. (C) RAC1 and p-PYK2 protein expression in HUVECs from M1 macrophage-cocultured system treated with VLA4 inhibitor CDP323 ( $n=3$ ). (D) RAC1 and p-PYK2 expression in HUVECs from M1 macrophage-cocultured system treated with VCAM1 inhibitor K-7174 $(n=3)$. (E) Flow cytometric analysis of ROS levels in HUVECs from M1 macrophage-cocultured system treated with RAC1 inhibitor NSC23766 $(n=3)$. (F and G) p-PYK2 expression in HUVECs from M1 macrophage-cocultured system treated with NAC (F) or and M2 macrophage-cocultured system treated with $\mathrm{H}_{2} \mathrm{O}_{2}(\mathbf{C})(n=3)$. Results represent 3 independent experiments. Results are shown as mean $\pm \mathrm{SD}$. ${ }^{*} P<0.05$; ${ }^{* *} P<0.01$; ${ }^{* *} P<0.001$; ${ }^{* * *} P<0.0001$, Student's $t$ test (C-G) and 1-way ANOVA (A).

shown in Figure 5E. Compared with the isotype control group, ascites volume in the PS/2 treatment group was abrogated by more than $60.9 \%$ (Figure $5, \mathrm{~F}$ and $\mathrm{G}$ ). We also examined the alterations of the RAC1/ROS/p-PYK2 cascade in the endothelium in HM-1 tumors upon PS/2 treatment. Consistently, PS/2 treatment resulted in fewer ROS-positive cells in ECs isolated from HM-1 tumors (Figure 5H). RAC1, p-PYK2, and p-VE-cad levels were also markedly reduced compared with those in controls by immunofluorescence staining (Figure 5, I-M). Taken together, these findings illustrate that VLA4 indeed plays a crucial role in macrophage-mediated permeability, which provides a therapeutic target for the treatment of ascites.
VLA4/VCAM1 expression is correlated with ascites volume. In order to verify our findings in the clinical setting, we first analyzed VLA4 expression in OC patient samples with or without signs of ascites that were found in the Kaplan-Meier Plotter database. Intriguingly, patients with low VLA4 expression survived much longer than patients expressing higher levels of VLA4 (Figure 6A). More importantly, the expression levels of VCAM1 or VLA4 in OC tissues were correlated with ascites volume in patients, as observed by immunohistochemical and immunofluorescence staining (Figure 6, B-E). However, the sVCAM1 expression detected in ascites of less than $500 \mathrm{ml}$ (few) was identical to that in ascites of more 
A

E

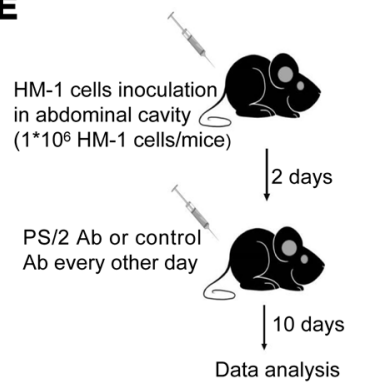

$\mathbf{B}$

$\mathbf{F}$

$\mathrm{PS} / 2$
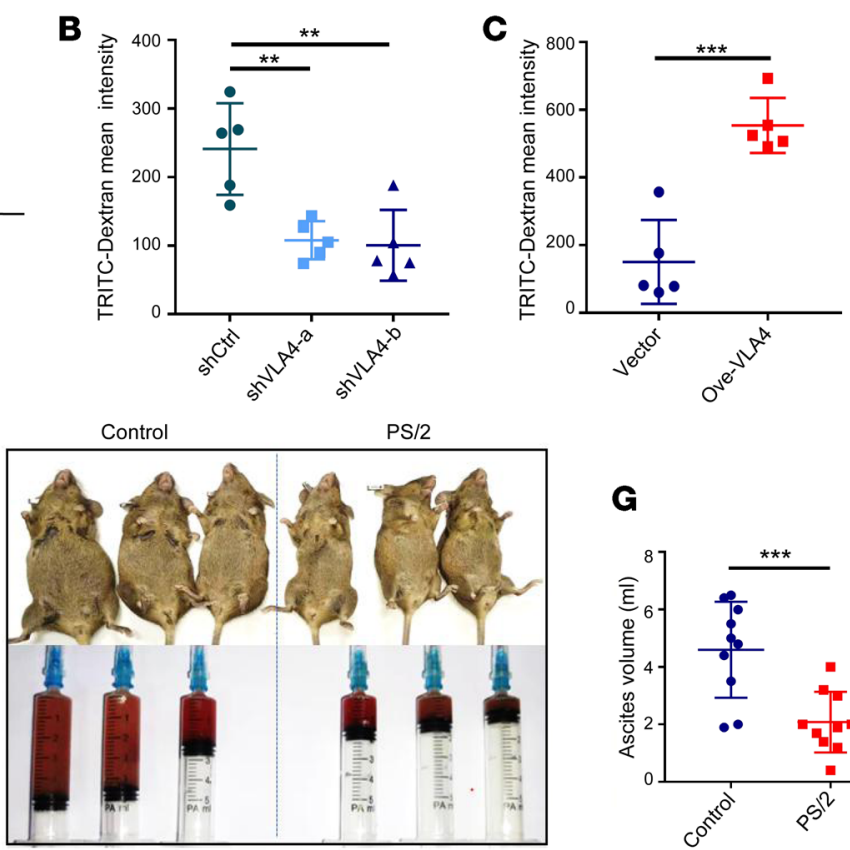

G

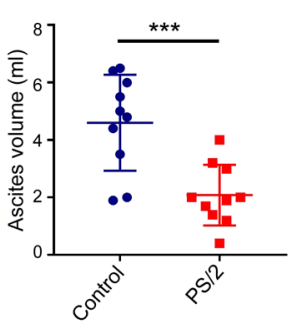

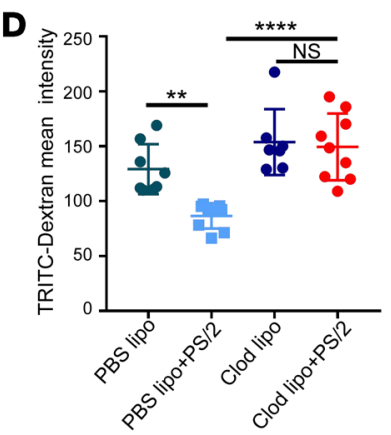

H

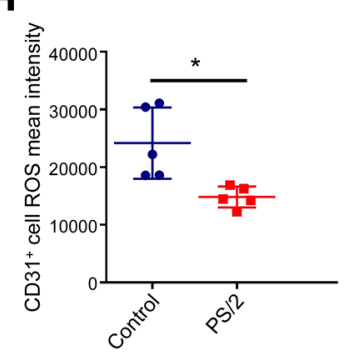

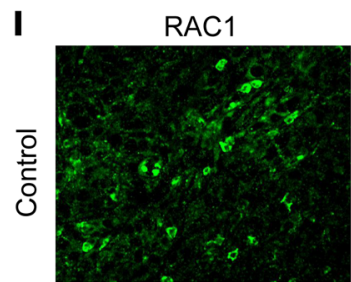
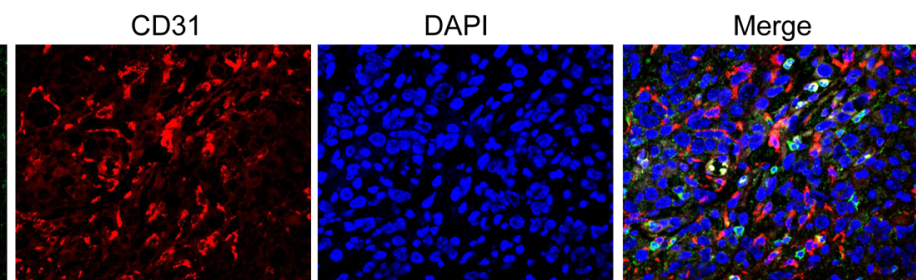

J
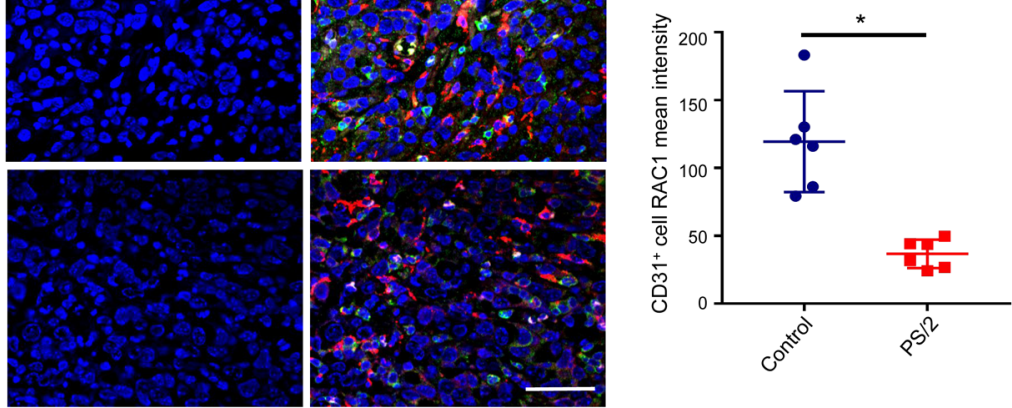

$\frac{2}{\mathrm{~N}}$
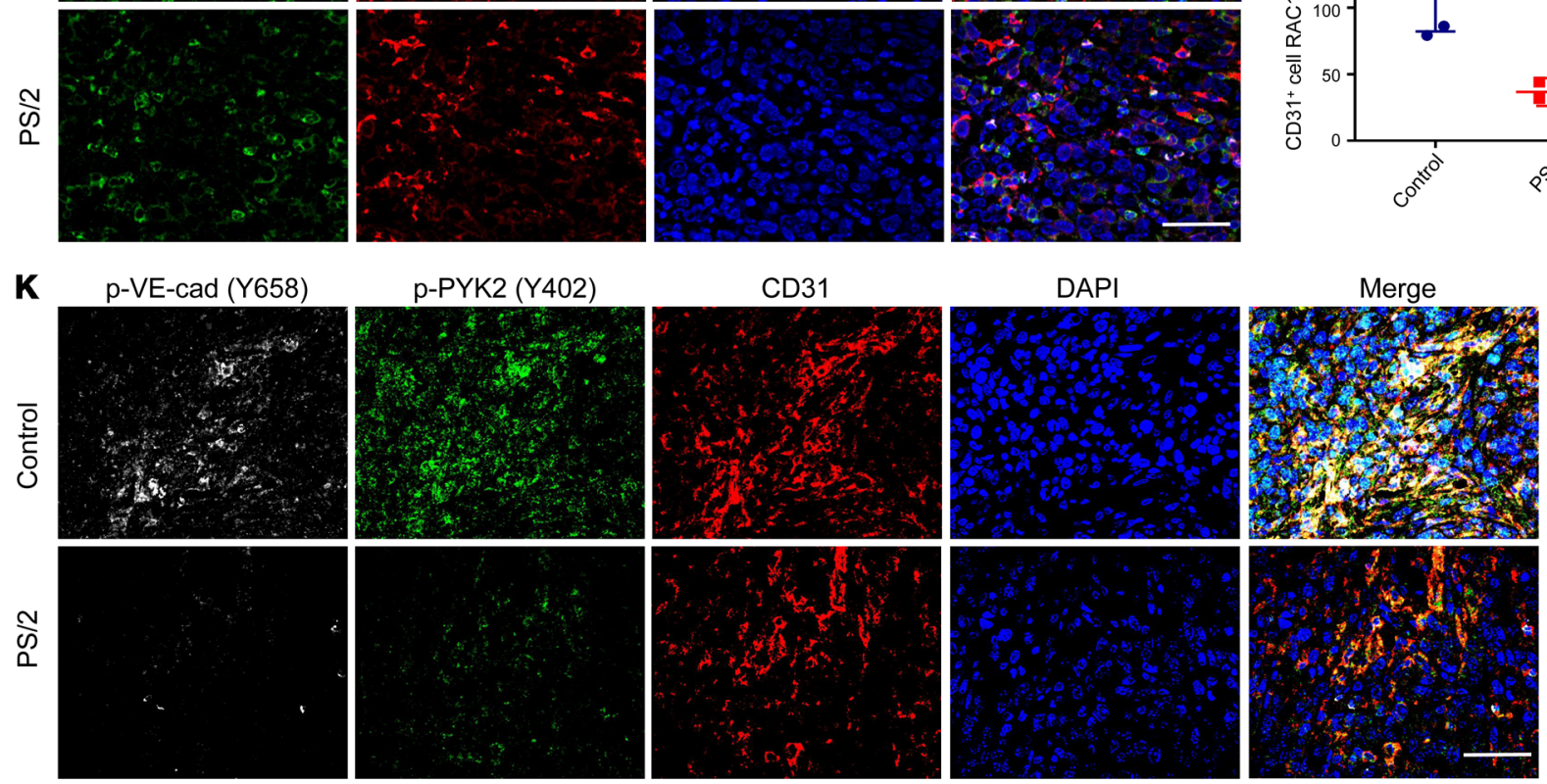

$\mathbf{L}$

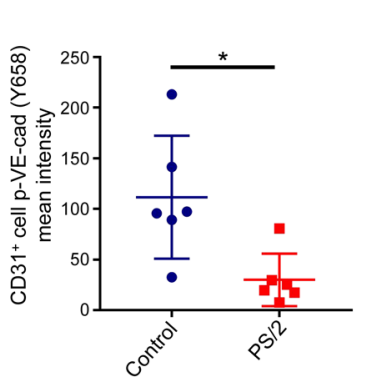

M

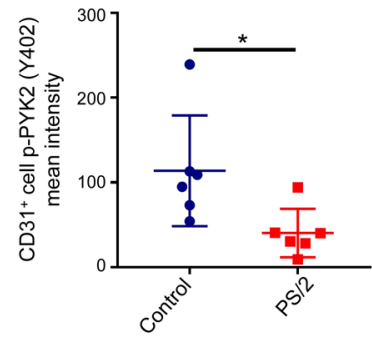


Figure 5. Targeting the VLA4/VCAM1 pathway reduces permeability and lessens ascites in vivo. (A) Schematic of experimental design for the permeability assay in vivo. Clod, clodronate liposomes; treated cell, RAW264.7 cells with VLA4 knockdown or overexpression. (B and C) Evaluation of peritoneal permeability 48 hours after injection of VLA4 knockdown (B) or overexpression (C) cells in the peritoneum of mice $(n=5)$. (D) Evaluation of peritoneal permeability 48 hours after injection of PS/2-treated cells in the peritoneum of mice treated with liposomes ( $n=8$ for PBS lipo group; $n=9$ for PBS lipo+PS/2 group; $n=7$ for Clod Lipo; $n=9$ for Clod lipo+PS/2). (E) Schematic of experimental design for treating ascites in $\mathrm{OC}$ animal. (F) Representative photographs of adult mice with $\mathrm{OC}$ sacrificed 10 days after PS/2 or lgC2b treatment and the ascites each mouse produced. (C) Ascites volume from $\mathrm{PS} / 2$ - or control antibody-treated OC mice 12 days after $\mathrm{HM}-1$ inoculation ( $n$ $=10)$. (H) ROS expression of ECs in tissue after treatment with PS/2 in mice with OC $(n=5)$. (I-M) Expression of RAC1 (I-J) and p-PYK2 and p-VE-cad (K-M) in murine OC tumors detected by immunofluorescence $(n=6)$. Scale bars: $50 \mu \mathrm{m}$. Results represent 3 independent experiments. Results are shown as mean $\pm \mathrm{SD}$. ${ }^{*} P<0.05$; ${ }^{* *} P<0.01$; ${ }^{* *} P<0.001$, ${ }^{* * *} P<0.0001$, 1-way ANOVA (B and $\mathbf{D}$ ) and Student's $t$ test (C, G, H, J, L, and $\mathbf{M})$.

than $1000 \mathrm{~mL}$ (massive; Supplemental Figure 6A). Furthermore, flow cytometry analysis showed that more macrophages expressed VLA4 in massive ascites (Figure 6F) and that macrophages from massive ascites expressed higher levels of VLA4 than those with fewer ascites (Supplemental Figure 6B). Interestingly, both patients and animals with massive ascites exhibited a lower ratio of M2 to M1 macrophages than those with fewer ascites (Figure $6 \mathrm{G}$ and Supplemental Figure $6 \mathrm{C}$ ).

To confirm that macrophages from massive ascites could indeed induce permeability, we isolated macrophages from patient and mouse ascites, cocultured them with ECs, and measured the barrier changes by dextran permeability assay and by electric cell impedance sensing (ECIS) equipment. Both assays validated our hypothesis (Figure 6, H and I, and Supplemental Figure 6D). Blocking either VLA4, VCAM1, or ROS rescued the compromised vascular barrier (Figure 6J and Supplemental Figure 6E). In summary, these results strongly suggest that the levels of VCAM1 and VLA4 positively correlate with ascites development in OC patients and may be used as therapeutic targets for ascites treatment.

VLA4/VCAM1 axis dictates permeability independently of the VEGF pathway. To further discern the role of the VEGF pathway in macrophage direct contact-mediated permeability, we used bevacizumab to block VEGF signaling. After adding bevacizumab to the coculture system, both permeability and p-VE-cad expression were significantly reduced in the coculture with M1 macrophages, but were not altered as much in the M2 macrophage-cocultured system (Figure 7, A and B), suggesting that the regulation of vascular permeability by M2 macrophages did not, at least not totally, depend on VEGF. Furthermore, there was no correlation between VEGF and macrophage VLA4 expression in ascites of OC patients (Supplemental Figure 7A). Therefore, we speculated that the 2 pathways worked separately in this system. To prove this point, we combined antibodies that block VEGF and VLA4, respectively, to treat the cocultures and obtained a synergistic effect in reducing permeability (Figure 7C). Next, we compared the effects of each individual and the combinatorial treatment in HM-1 animals. B-scan ultrasonography was conducted to measure the volume of ascites in the abdominal cavity. We observed that the volume of ascites was strikingly reduced in the combinatorial treatment group, although individual treatment was also effective and comparable to each other (Figure 7, D and E). The survival data also supported the above findings (Figure 7F).

Finally, we investigated the potential effect of blocking VLA4 on macrophage polarization and found that PS/2 treatment could significantly enhance M1-markers, such as IL6, NOS2, and IL12B, while significantly decreasing the levels of M2 markers, such as $A R G 1$ and CD206, in M1 macrophages (Figure 7G). For M2 macrophages, $\mathrm{PS} / 2$ treatment consistently induced the expression of $I L 6$, NOS2, and IL12B, which are often expressed by M1 macrophages and related to antitumor effect, but meanwhile decreased the levels of M2-markers, such as IL1O and ARG1 (Figure 7H). It should be noted that VEGF expression was not significantly changed in either group. These results indicate that PS/ 2 treatment may increase the tumor-killing capability of macrophages while exerting the barrier protection function. In summary, all the data suggest that M2 macrophages can regulate EC permeability in a pathway that is independent of VEGF and that anti-VLA4 therapy may be used as a therapeutical strategy to treat malignant ascites.

\section{Discussion}

Malignant ascites is observed in most terminal OCs, and it markedly contributes to mortality and poor quality of life. However, the formation and regulation of ascites are poorly understood, and targeted therapy to treat ascites has not been developed. In this study, we demonstrate that M2 macrophages could tune down the VCAM1 expression in the endothelium and suppress the RAC1/ ROS/p-PYK2/p-VE-cad downstream cascade, thus enhancing vascular integrity. Targeting the VLA4/VCAM1 axis could attenuate vascular permeability both in vitro and in vivo and, more importantly, inhibit ascites formation in OC animal models. Our findings have uncovered a molecular mechanism of M2 macrophage-mediated vascular permeability and provided targets for treating ascites in $\mathrm{OC}$ patients.

Macrophages actively participate in the maintenance of vascular homeostasis. Since we reported that macrophage depletion led to the loss of vascular integrity (12), several studies have observed similar effects in various organs. For example, when macrophages are depleted in stria vascularis of the cochlea, tight junctions between ECs become unstable, resulting in increased vessel permeability and, ultimately, the loss of hearing (21). Similarly, in the blood-brain barrier (BBB), when ECs isolated from the mouse brain were cocultured with macrophages, the permeability was markedly reduced (22). However, the role of macrophage-mediated permeability in OC ascites formation has not been elucidated. Moreover, tumor-associated macrophages (TAMs) are believed to be more M2 like; this is detrimental to health because they promote tumor growth and angiogenesis (23). Although a high ratio of M1/M2 macrophages predicts an improved prognosis, as reported in an OC study (24), our data suggest that a high M1/M2 ratio is also associated with more ascites in both murine $\mathrm{OC}$ models and OC patients, likely due to loss of the protection of the M2 macrophages in the vascular barrier. These findings uncovered a role for M2 macrophages or TAMs in OC development. Given that ascites can be detrimental to the quality of life and the progression of the disease, eliminating macrophages through techniques such as treatment with anti-CSF1R (25) or through the reprogramming of 
A

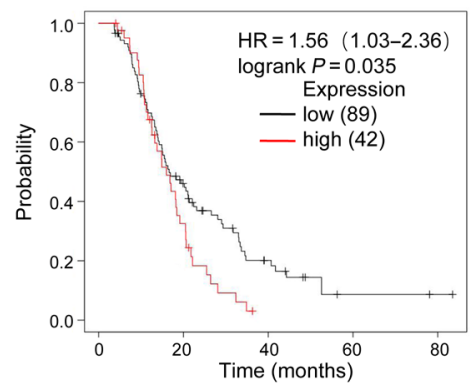

B

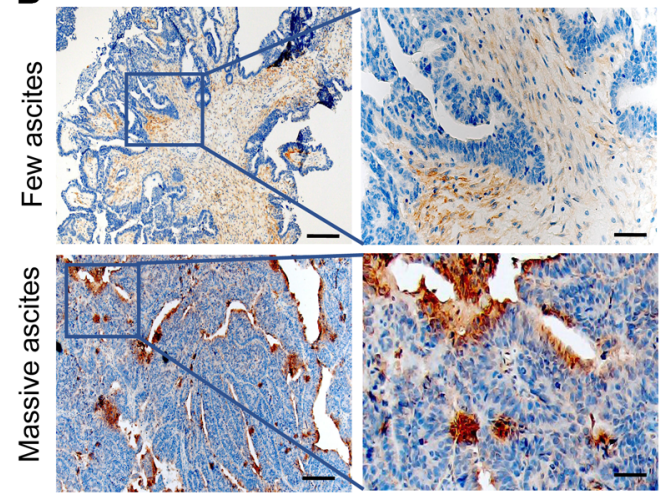

C

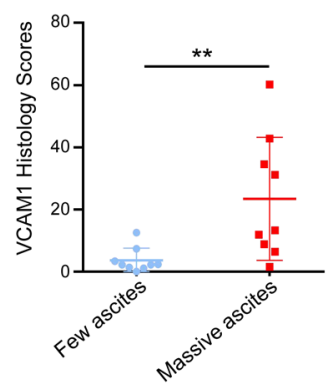

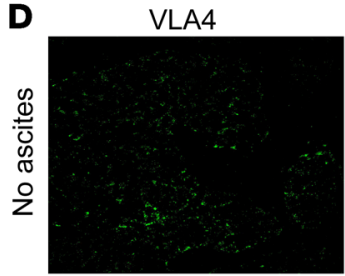
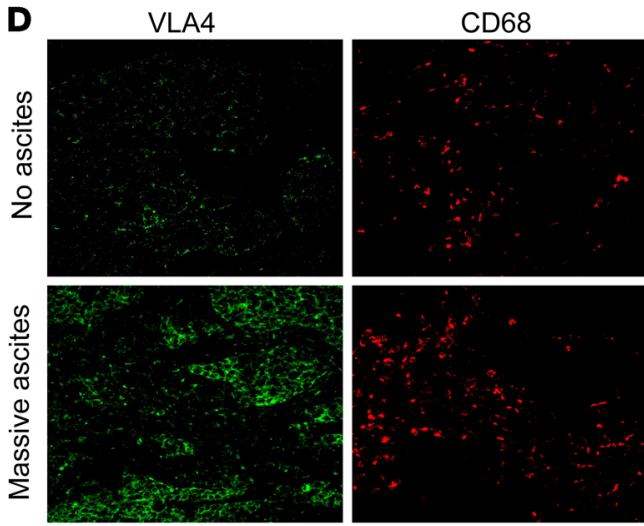

$\mathbf{F}$

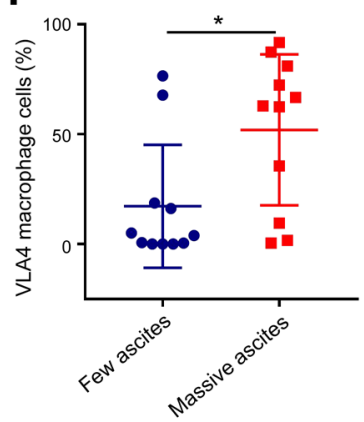

I

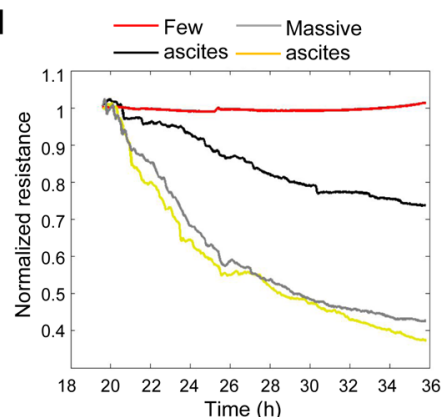

G

J
DAPI
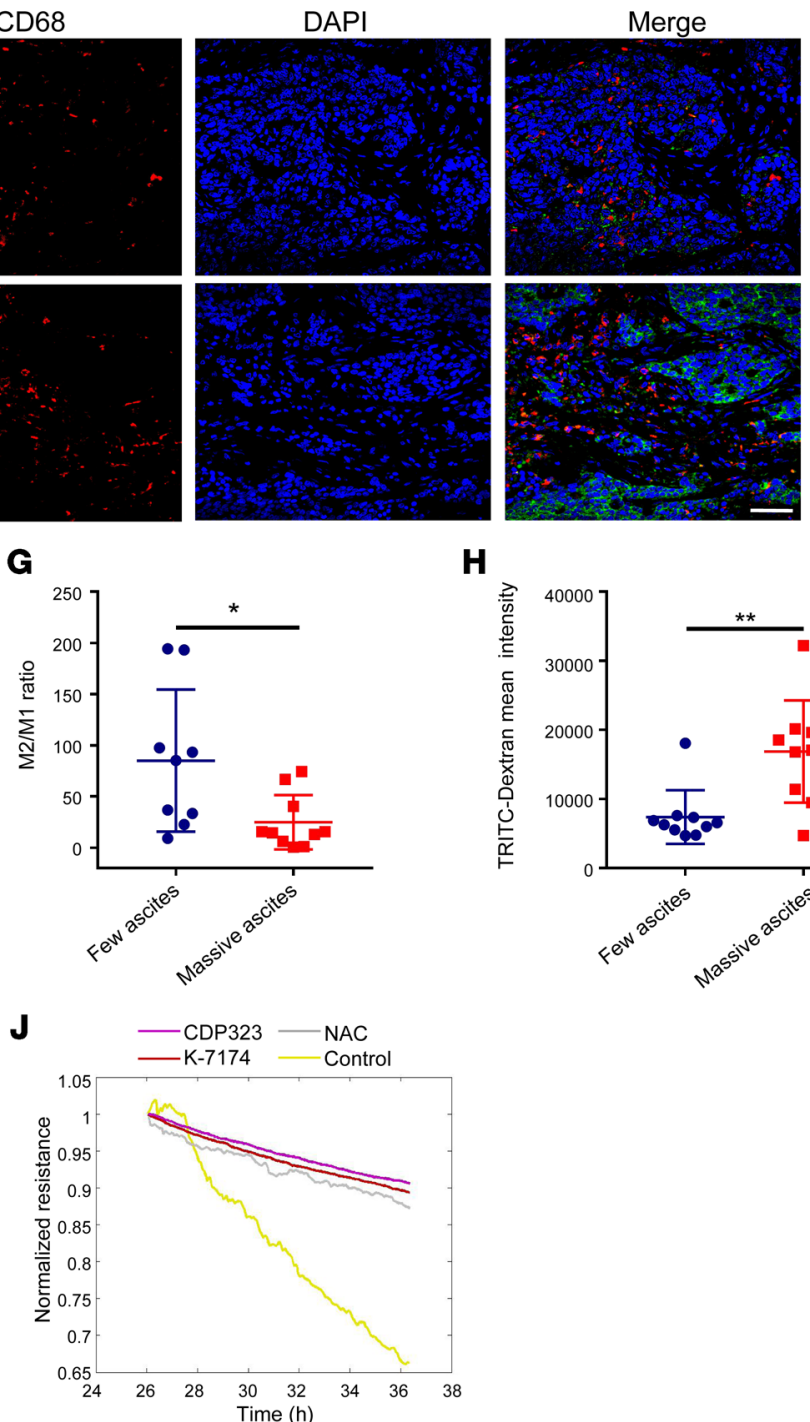

H

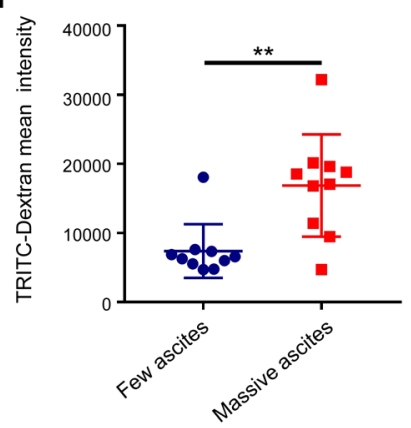

E

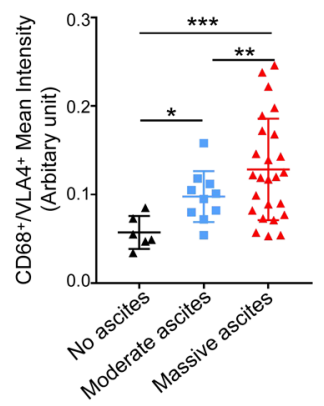

Figure 6. VLA4/VCAM1 expression is correlated with ascites volume. (A) Kaplan-Meier Plotter analysis showed that high VLA4 expression is correlated with shorter overall survival. (B and C) Analysis of VCAM1 expression in OC tissues from patients with few ascites (less than 500 ml) or massive ascites (more than $1000 \mathrm{~mL}$ ) based on immunohistochemistry results $(n=9)$. Scale bars: $200 \mu \mathrm{m}$ (left); $50 \mu \mathrm{m}$ (right). (D) Representative immunofluorescence images of VLA4 expression in human OC tumors from patients with few ascites or massive ascites $(n=10)$. Green, VLA4; red, CD68; blue, nucleus. Scale bar: $50 \mu \mathrm{m}$. (E) Correlation between ascites volume and VLA4 expression in macrophages of OC tissue. Few ascites (less than 500 ml); moderate ascites $(500 \mathrm{ml}-1000 \mathrm{ml}$ ); massive ascites (more than $1000 \mathrm{~mL}$ ). (F) Percentage of VLA4-expressing macrophages in different $0 \mathrm{C}$ ascites ( $n=11$ ). (G) Ratio of M2 to $\mathrm{M} 1$ macrophages in OC ascites ( $n=9$ for few ascites group, $n=10$ for massive ascites group). (H) Permeability analysis using macrophages isolated from OC patient ascites detected by TRITC-Dextran assay $(n=10)$. (I) Permeability assay using macrophages isolated from OC patient ascites detected by ECIS equipment. (J) Permeability assay of macrophages isolated from OC patient ascites cocultured with HUVECs with CDP323, K-7174, NAC, or control treatment detected by ECIS. Results represent 3 independent experiments. Results are shown as the mean \pm SD. ${ }^{*} P<0.05 ;{ }^{*} P<0.01 ;{ }^{*}{ }^{*} P<0.001,1-$ way ANOVA (E) and Student's $t$ test (C and F-H). 
A

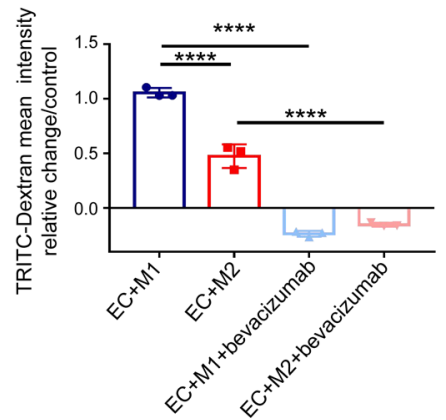

C

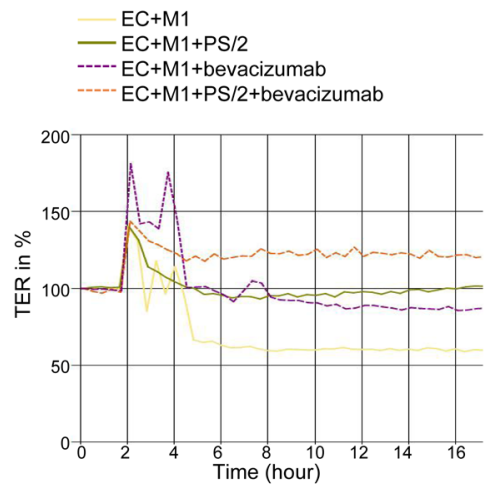

$\mathbf{F}$

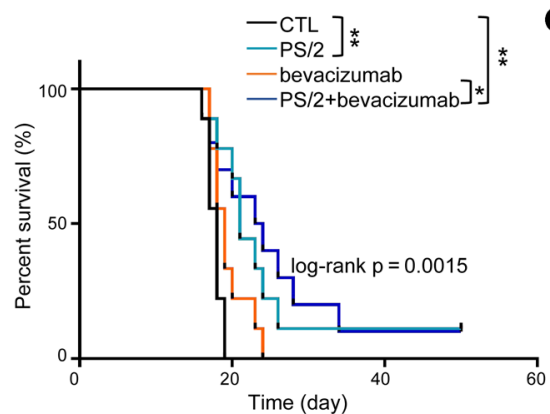

B
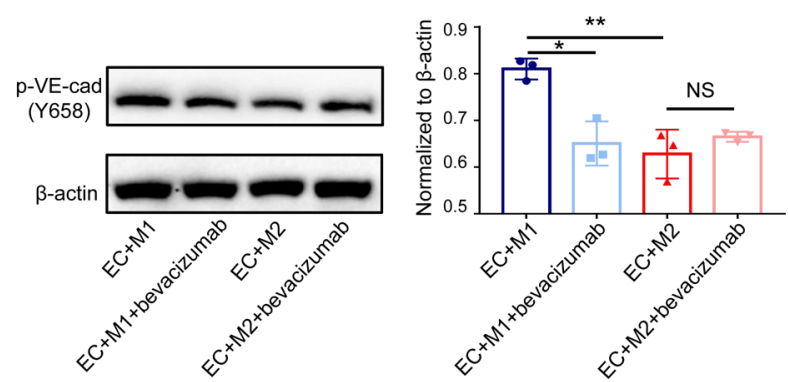

D
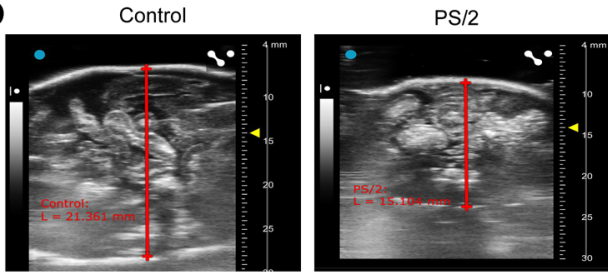

bevacizumab

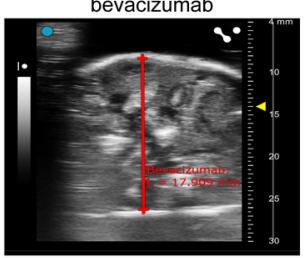

PS/2+bevacizumab

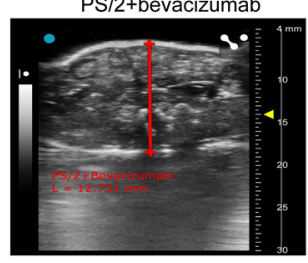

E

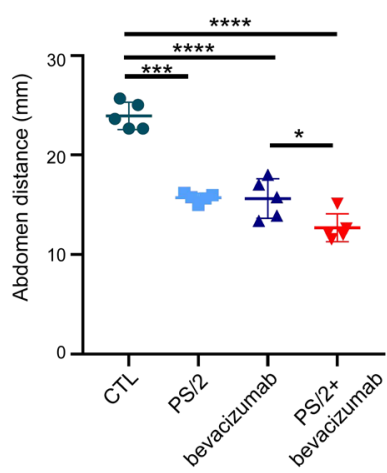

G
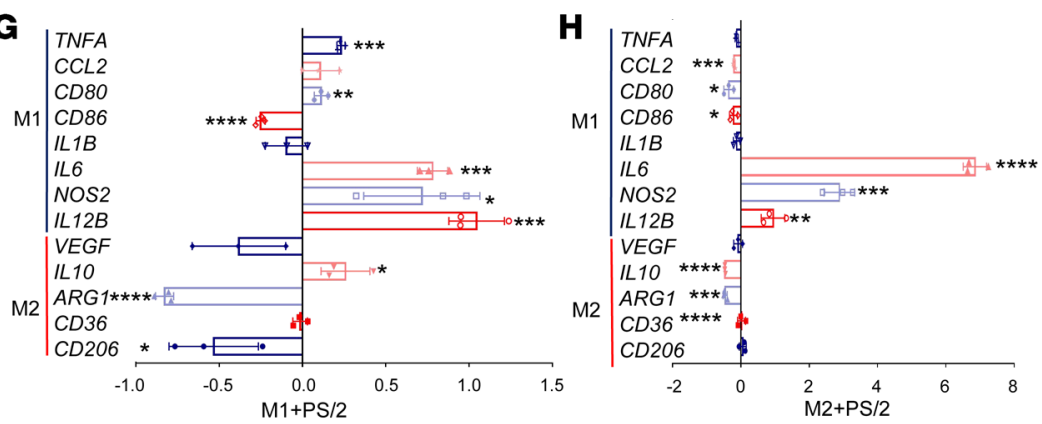

Figure 7. VLA4/VCAM1 axis mediates permeability separately from the VEGF pathway. (A) TRITC-dextran permeability in HUVECs cocultured with M1 or M2 macrophages before or after treatment with bevacizumab $(n=3)$. (B) p-VE-cad expression in HUVECs from M1 macrophage-cocultured system or M2 macrophage-cocultured system after treated with bevacizumab $(n=3)$. (C) Permeability assay using M1 macrophage coculture treated with PS/2, bevacizumab, or combination of PS/2 and bevacizumab detected by the automated system (cellZscope). (D and E) Ascites volume detected by B mode ultrasonography after i.p. injection in mice with PS/2, bevacizumab, or both $(n=5)$. (F) Kaplan-Meier survival curves of mice after i.p. injection with PS/2, bevacizumab, or both $(n=9)$. ( $(\mathbf{G}$ and $\mathbf{H})$ Fold change of gene expression in M1 macrophages $(\mathbf{C})$ or M2 macrophages (H) treated with PS/2 compared with M1 or M2 macrophages alone $(n=3)$. Data represent 3 independent experiments. Results are shown as mean $\pm \mathrm{SD}$. ${ }^{*} P<0.05 ;{ }^{* *} P<0.01 ;{ }^{* *} P<0.001 ;{ }^{* * * *} P$ $<0.0001,1$-way ANOVA (A, B, and $\mathbf{E}$ ) and Student's $t$ test ( $\mathbf{G}$ and $\mathbf{H})$. The Kaplan-Meier analysis log-rank test was used to estimate the event-free survival curve between the groups $(\mathbf{F})$.

M2 to M1 macrophages using thiazolidinediones (TZDs) (26) may not be the wisest strategy in terms of prolonging the overall survival and quality of life of OC patients.

Direct interactions between macrophages and ECs are critical in pathological conditions, such as inflammation and atherosclerosis. For instance, M1 macrophage-induced inflammatory responses involve monocytes tethering to the endothelium using selectins and subsequently monocytes using VLA4, also known as $\alpha_{4} \beta_{1}$ integrin, to engage VCAM1 or intercellular adhesion molecule 1 (ICAM-1) on the endothelium to form a firm adhesion; this then triggers downstream RAC1/ROS/p-PYK2/p-VE-cad signaling and ultimately leads to hyperpermeability (27-31). Interestingly, we found that M2 macrophages used the same pathway, but in reverse to achieve a different, or rather opposite, effect. Our data showed that M2 macrophages hampered VCAM1 expression in the endothelium and tuned down RAC1/ROS/p-PYK2/p-VE-cad signaling, leading to an enhanced vascular barrier. We think this is an efficient way for macrophages to regulate vascular permeability in a dynamic environment.

Malignant ascites presents a considerable clinical challenge to the management of $\mathrm{OC}$, yet there is a lack of treatment options at present. Diuretics are frequently used in the treatment of ascites arisen from nonmalignant disease (2). However, no solid evidence has been shown to date for the efficacy of diuretics in malignant asci- 
tes $(32,33)$. Alternatively, i.p. administration of bleomycin, mitoxantrone, or fluorouracil in combination with cisplatin has been reported to improve the condition of ascites (34-37). Nonetheless, the specificity for antiascites has not been fully evaluated, and the antiascites mechanism has not been discussed. Another popular strategy for controlling ascites is to inhibit VEGF signaling, since VEGF is a well-known vascular permeator secreted by tumor cells and immune cells. Therefore, bevacizumab, an antibody that functionally blocks VEGF, has seen an increase in clinical use. Yet resistance to antiVEGF therapy remains a key problem, and efforts need to be made to understand the underlying mechanisms (38). Here, we describe a specific and potent ascites-targeting antibody, PS/2, that blocks VLA4 and downstream signaling and shows substantial efficacy in abolishing ascites development. Since the VLA4/VCAM1 pathway is distinct from the VEGF signaling pathway regarding the regulation of vascular permeability, when bevacizumab was administered in our coculture system, M2-mediated endothelial permeability was not altered as much as that of M1. In addition, when combining PS/2 and bevacizumab, we obtained a synergistic antipermeability effect and extended survival rate in OC-bearing mice. These results suggest that the M2 macrophage-mediated VLA4/VCAM1 pathway can regulate EC permeability independently of the VEGF pathway.

In summary, we found that VLA4 in M2 macrophages and VCAM1 in ECs were both downregulated upon direct engagement of these 2 types of cells, which suppressed the downstream RAC1/ ROS/p-PYK2/p-VE-cad pathway, ultimately leading to enhanced vascular integrity. Our results provide an improved understanding of the key role that macrophages play in the regulation of vasculature under the context of OC and reveal a therapeutic target for the treatment of malignant ascites.

\section{Methods}

Patients and samples. Fresh tumor, nontumor ovarian tissues, and ascites were collected from 32 patients diagnosed with high-grade serous ovarian carcinoma at the Fifth Affiliated Hospital of Sun Yat-sen University. Ascites (taken on day of surgery) from OC patients who underwent surgical resection were used for isolating macrophages. The clinical characteristics of all patients in 2 cohorts are listed in Supplemental Table 1.

Cell lines and cell culture. HUVECs, a murine EC line (C166), and a murine macrophage cell line (RAW264.7) were obtained from ATCC. A human macrophage cell line (THP-1) was a gift from Dong-Ming Kuang (Sun Yat-sen University). Cells from a murine ovarian carcinoma cell line, OV2944-HM-1 (HM-1), were a gift from Nelson Teng (Stanford University, Stanford, California, USA). The cells were authenticated by short tandem repeat profiling and were confirmed to be mycoplasma free before use. HUVECs were cultured in ECM (ScienCell) supplemented with 5\% FBS, 1\% EC growth supplement (ECGS), $100 \mathrm{U} /$ $\mathrm{ml}$ penicillin, and $100 \mu \mathrm{g} / \mathrm{ml}$ streptomycin. THP- 1 cells and HM-1 cells were cultured in RPMI 1640 medium supplemented with 10\% FBS, 100 $\mathrm{U} / \mathrm{ml}$ penicillin, and $100 \mu \mathrm{g} / \mathrm{ml}$ streptomycin. C166 and RAW264.7 cells were cultured in DMEM (Invitrogen) supplemented with 10\% FBS, 100 $\mathrm{U} / \mathrm{ml}$ penicillin, and $100 \mu \mathrm{g} / \mathrm{ml}$ streptomycin. All cells were maintained in a humidified incubator with $5 \% \mathrm{CO}_{2}$ that was at $37^{\circ} \mathrm{C}$.

Western blot. The protein samples determined by BCA assay (Beyotime Biotechnology) were separated by $4 \%-12 \%$ SDS-PAGE and transferred onto PVDF membrane. The primary antibodies used in this study are listed in Supplemental Table 2.
Permeability assay. HUVECs $\left(1 \times 10^{5}\right.$ cells $)$ were seeded on top of a Transwell insert in 24-well Transwell chambers and were cultured for 24 hours until reaching confluence. THP- 1 cells $\left(5 \times 10^{4}\right)$ polarized to different subtypes of macrophages were seeded on endothelial monolayers for another 24 hours. The samples were randomly divided into several groups: control group, CDP323-treated group, PS/2-treated group, THIO019-treated group, K-7174-treated group, NAC-treated group, and $\mathrm{H}_{2} \mathrm{O}_{2}$-treated group. The lower compartments were filled with medium alone (control group), medium containing CDP323 (2 $\mu \mathrm{mol} / \mathrm{L}$; CDP323 group), PS/2 ( $2 \mu \mathrm{mol} / \mathrm{L}, \mathrm{PS} / 2$ group), THIO019 (2 $\mu \mathrm{mol} / \mathrm{L}$, THIO019 group), K-7174 (2 $\mu \mathrm{mol} / \mathrm{L}, \mathrm{K}-7174$ group), NAC (5 $\mu \mathrm{mol} / \mathrm{L}, \mathrm{NAC}$ group $)$, or $\mathrm{H}_{2} \mathrm{O}_{2}\left(2 \mu \mathrm{mol} / \mathrm{L}, \mathrm{H}_{2} \mathrm{O}_{2}\right.$ group $)$. After 24 hours incubation, $100 \mu \mathrm{l}$ of PBS was used to wash the chambers twice. Then $500 \mu \mathrm{l}$ of PBS was added to the lower layer, and $200 \mu \mathrm{l}$ of PBS containing TRITC-dextran $(2 \mathrm{mg} / \mathrm{ml})$ was added to the upper layer. The entire transwell chamber was incubated for 3 hours at $37^{\circ} \mathrm{C}$. The PBS in the lower compartments was collected and analyzed in a fluorescence spectrophotometer (Synergy HTX, BIOTEK) with excitation and emission wavelengths of $530 \mathrm{~nm}$ and $540 \mathrm{~nm}$, respectively.

Alternatively, HUVECs were seeded onto 24-well Transwell and treated with chemicals as indicated. Then the barrier function was measured with the automated cell monitoring cellZscope system (NanoAnalytics) for 24 hours in a $\mathrm{CO}_{2}(5 \%)$ incubator at $37^{\circ} \mathrm{C}$.

ROS level measurement. ROS levels were measured with the dichlorodihydrofluorescein diacetate (DCFH-DA) assay (Beyotime Institute of Biotechnology, Nantong, China) according to the manufacturer's instructions. Briefly, the cells were resuspended in PBS and then were stained with $10 \mu \mathrm{M}$ DCFH-DA for 20 minutes at 37 ${ }^{\circ} \mathrm{C}$ in the dark. After washing with PBS, the samples were detected by flow cytometry with an excitation wavelength of $488 \mathrm{~nm}$ and an emission wavelength of $525 \mathrm{~nm}$.

Generation of lentiviral particles and cell transduction. VLA4 knockdown or stable overexpression in RAW264.7 cells was established by infecting cells with a lentiviral vector (U6-sh-VLA4-EGFP-IRES-puromycin) purchased from GeneCopoeia. RAW264.7 cells were infected with lentivirus using $0.8 \mu \mathrm{g} / \mathrm{ml}$ polybrene. Stable clones were selected after 2 weeks using $1 \mu \mathrm{g} / \mathrm{ml}$ puromycin. The efficiency of RNA interference was assessed via qRT-PCR and Western blot.

RNA interference. HUVECs were transfected for 24 hours with a nontargeting shRNA or a VCAM1 shRNA using Lipofectamine 3000 (Invitrogen; Thermo Fisher Scientific) and then were assayed 72 hours after transfection. Negative control shRNA (shRNA-control) and the empty vector ( $\mathrm{pEZ}-\mathrm{Vec}$ ) were provided as the control of shRNA-VCAM1 and pEZVCAM1 experiments, respectively. A plasmid targeting VLA4 (pEZ-VLA4, GenePharma) was used to upregulate VLA4 in cells. The constructs $(1.5 \mu \mathrm{g})$ were then transfected into $1 \times 10^{6} / \mathrm{ml}$ THP-1 cells (ATCC) using Lipofectamine 3000 (Invitrogen; Thermo Fisher Scientific Inc.) according to the manufacturer's instructions, and then they were assayed 72 hours after transfection.

Immunofluorescence staining. Coculture cells were seeded on chamber slides and cultured for 24 hours. Cells were washed with PBS, fixed with precooled paraformaldehyde for 10 minutes at $4^{\circ} \mathrm{C}$, permeabilized with $1 \%$ Triton X-100 (Solarbio Life Science) in PBS for 15 minutes, incubated in blocking solution for 1 hour at room temperature, and incubated with specific primary antibodies overnight at $4^{\circ} \mathrm{C}$. Then the samples were washed 3 times with PBS, incubated with secondary antibodies for 1 hour at room temperature in a dark 
place, washed 3 times with PBS, and incubated for 10 minutes in the dark with Fluoroshield Mounting Medium with DAPI. Images were acquired using confocal microscope.

IHC staining. Samples of OC tissues were acquired during surgery at the Fifth Affiliated Hospital of Sun Yat-sen University. Tissues were fixed in $10 \%$ neutral-buffered formalin overnight, dehydrated using increasing gradient concentrations, and embedded in low-melting point paraffin. Continuous $4 \mu \mathrm{m}$ thick tissue sections were cut and fixed onto silicified slides. Immunohistochemistry was carried out using the streptavidin/peroxidase-conjugated method. Briefly, each tissue section was deparaffinized, rehydrated, immersed in antigen retrieval solution, boiled at $100^{\circ} \mathrm{C}$ for 10 minutes, and incubated with a peroxidase inhibitor. Then nonspecific binding was blocked with normal goat serum, and tissue sections were incubated overnight at $4^{\circ} \mathrm{C}$ with the following primary antibodies: anti-VCAM1 (Abcam). All antibodies were used at a 1:50 dilution.

Animal experiments. Animals were maintained under pathogen-free conditions in the Guangdong Provincial Key Laboratory of Biomedical Imaging. Five groups of 5 BALB/c female mice (Vital River Laboratory Animal Technology Co.) aged 4 to 6 weeks were injected with clodronate and control liposomes were injected via i.p. injection at $200 \mu \mathrm{l} /$ mouse on day 1 . On day 3, liposomes were injected again following the same method. Macrophages with VLA4 knocked down, overexpressed, or unchanged $\left(10^{6}\right.$ cells in $\left.100 \mathrm{ul} \mathrm{PBS}\right)$ were delivered via i.p. injection. Two days later, $200 \mu \mathrm{l}$ TRITC-dextran (Invitrogen) was injected i.v. into each mouse. After specific time intervals, animals were euthanized and $1 \mathrm{ml}$ of PBS was injected i.p. to recover the peritoneal macrophages and liquid in the peritoneal cavity. The solution was then spun down, and from this solution, the supernatant was taken for fluorescence intensity quantification.

The B6C3F1 mouse, aged 6 to 8 weeks,was produced as a cross between a male $\mathrm{C} 3 \mathrm{H}$ mouse and a female $\mathrm{C} 57 \mathrm{BL} / 6$ mouse. For HM-1 animal models, $10^{6} \mathrm{HM} 1$ cells were injected into the peritoneal cavity via a left lower abdominal wall injection. Mice were treated with PS/2 $(3 \mathrm{mg} / \mathrm{kg})$ every other day at 2 days following injection. Mice were sacrificed at 2 weeks using $\mathrm{CO}_{2}$ gas per institutional protocols.

Statistics. The results are presented as mean \pm SD of at least 3 independent experiments. GraphPad Prism software (version 5.0) was used for all statistical analyses. Differences between groups were evaluated using Student's $t$ tests when only 2 groups were analyzed or by 1 -way ANOVA when more than 2 groups were compared. Pearson's correlation was performed to analyze the correlation between protein expression of VLA4 and VEGF. $P<0.05$ was considered statistically significant.

Study approval. All animal experiments were performed with the approval of the Experimental Animal Ethics Committee of the Fifth
Affiliated Hospital of Sun Yat-sen University and in accordance with the Guidelines for the Ethical Review of Laboratory Animal Welfare (GB/ T35892-2018) and institutional ethical guidelines for animal experiments developed by the Fifth Affiliated Hospital of Sun Yat-sen University. All human samples were anonymously coded in accordance with local ethical guidelines (as stipulated by the Declaration of Helsinki). Written, informed consent was obtained from patients, and the protocol was approved by the Medical Ethics Committee of the Fifth Affiliated Hospital of Sun Yat-sen University (2018-K181-1).

\section{Author contributions}

SZ, BX, LW, FW, CL, and $\mathrm{HH}$ conceived and designed the study. SZ, BX, LW, HY, and HZ developed the methodology. SZ, BX, LW, $\mathrm{HY}, \mathrm{HZ}$, and YC acquired data. SZ, LW, HZ, and HH analyzed and interpreted data (e.g., performed statistical analysis, biostatistics, and computational analysis). SZ, BX, LW, CL, and HH wrote and reviewed the paper and/or revised the manuscript. SZ, BX, LW, $\mathrm{HY}, \mathrm{FW}, \mathrm{CL}$, and $\mathrm{HH}$ provided administrative, technical, or material support (i.e., reporting or organizing data, constructing databases). FW, CL, and HH supervised the study. SZ, BX, LW, and HY provided equal contributions in conceiving the overall study, performing experiments and conducting statistical analysis. HY was responsible for the clinical diagnosis and follow-up of the patients. $\mathrm{CL}$ and $\mathrm{HH}$ provided equal contributions in designing and overseeing the overall study and writing and reviewing the manuscript. The order of the first or last authors reflects the leadership exerted in the study.

\section{Acknowledgments}

The authors acknowledge Luisa Iruela-Arispe (Northwestern University, Chicago, Illinois, USA) and Nelson Teng and Oliver Dorigo (Stanford University) for their technical and material support and suggestions on the manuscript and Man Li for statistical consultation. This work was supported by project grants from the National Natural Science Foundation of China (grants 31741068 and 81872113) and support from Sun Yat-sen University (grant 18ykzd02).

Address correspondence to: Huanhuan He, Guangdong Provincial Key Laboratory of Biomedical Imaging, The Fifth Affiliated Hospital of Sun Yat-sen University, No. 52 Mei Hua Dong Road, Zhuhai 519000, China. Phone: 86.756.252.6143; Email: hehh23@ mail.sysu.edu.cn. Or to: Changqing Liu, Department of Gynaecology, The Fifth Affiliated Hospital of Sun Yat-sen University, No. 52 Mei Hua Dong Road, Zhuhai 519000, China. Phone: 86.1.392.694.7846; Email: 13926947846@sina.cn.
1. Miller KD, et al. Cancer treatment and survivorship statistics, 2016. CA Cancer J Clin. 2016;66(4):271-289.

2. Kipps E, et al. Meeting the challenge of ascites in ovarian cancer: new avenues for therapy and research. Nat Rev Cancer. 2013;13(4):273-282.

3. Ayantunde AA, Parsons SL. Pattern and prognostic factors in patients with malignant ascites: a retrospective study. Ann Oncol. 2007;18(5):945-949.

4. Parsons SL, et al. Malignant ascites: a 2-year review from a teaching hospital. Eur J Surg Oncol. 1996;22(3):237-239.
5. Fleming ND, et al. Indwelling catheters for the management of refractory malignant ascites: a systematic literature overview and retrospective chart review. JPain Symptom Manage. 2009;38(3):341-349.

6. Tan DSP, et al. Mechanisms of transcoelomic metastasis in ovarian cancer. Lancet Oncol. 2006;7(11):925-934.

7. Ahmed N, Stenvers KL. Getting to know ovarian cancer ascites: opportunities for targeted therapy-based translational research. Front Oncol. 2013;3:256.

8. Cavazzoni E, et al. Malignant ascites: patho- physiology and treatment. Int J Clin Oncol. 2013;18(1):1-9.

9. Ono S, et al. Regulation of blood vascular permeability in the skin. Inflamm Regen. 2017;37:11.

10. Fushida S, et al. VEGF is a target molecule for peritoneal metastasis and malignant ascites in gastric cancer: prognostic significance of VEGF in ascites and efficacy of anti-VEGF monoclonal antibody. Onco Targets Ther. 2013;6:1445-1451.

11. Bamias A, et al. Correlation of NK T-like CD3+CD56+ cells and CD4+CD25+(hi) regulatory $\mathrm{T}$ cells with VEGF and TNF alpha in ascites 
from advanced ovarian cancer: Association with platinum resistance and prognosis in patients receiving first-line, platinum-based chemotherapy. Gynecol Oncol. 2008;108(2):421-427.

12. $\mathrm{He} \mathrm{H}$, et al. Perivascular macrophages limit permeability. Arterioscler Thromb Vasc Biol. 2016;36(11):2203-2212.

13. Osada T, et al. Interendothelial claudin-5 expression depends on cerebral endothelial cell-matrix adhesion by $\beta(1)$-integrins. J Cereb Blood Flow Metab. 2011;31(10):1972-1985.

14. Obermeier B, et al. Development, maintenance and disruption of the blood-brain barrier. Nat Med. 2013;19(12):1584-1596.

15. Malinin NL, et al. Integrin signaling in vascular function. Curr Opin Hematol. 2012;19(3):206-211.

16. Vockel M, Vestweber D. How T cells trigger the dissociation of the endothelial receptor phosphatase VE-PTP from VE-cadherin. Blood. 2013;122(14):2512-2522.

17. Kevil CG, et al. Expression of zonula occludens and adherens junctional proteins in human venous and arterial endothelial cells: role of occludin in endothelial solute barriers. Microcirculation. 1998;5(2-3):197-210.

18. Sun $\mathrm{HJ}$, et al. Role of endothelial dysfunction in cardiovascular diseases: the link between inflammation and hydrogen sulfide. Front Pharmacol. 2020;10:1568.

19. Wu JR, et al. Hydrogen peroxide inducible clone5 sustains NADPH oxidase-dependent reactive oxygen species-c-jun N-terminal kinase signaling in hepatocellular carcinoma. Oncogenesis. 2019;8(8):40.
20. Daou GB, Srivastava AK. Reactive oxygen species mediate Endothelin-1-induced activation of ERK1/2, PKB, and Pyk2 signaling, as well as protein synthesis, in vascular smooth muscle cells. Free Radic Biol Med. 2004;37(2):208-215.

21. Okano T, Kishimoto I. Csf1 signaling regulates maintenance of resident macrophages and bone formation in the mouse cochlea. Front Neurol. 2019;10:1244.

22. Rodrigues SF, Granger DN. Blood cells and endothelial barrier function. Tissue Barriers. 2015;3(1-2):e978720.

23. Mantovani A, et al. Macrophage polarization: tumor-associated macrophages as a paradigm for polarized M2 mononuclear phagocytes. Trends Immunol. 2002;23(11):549-555.

24. Zhang MY, et al. A high M1/M2 ratio of tumor-associated macrophages is associated with extended survival in ovarian cancer patients. JOvarian Res. 2014;7:19.

25. Ries $\mathrm{CH}$, et al. Targeting tumor-associated macrophages with anti-CSF-1R antibody reveals a strategy for cancer therapy. Cancer Cell. 2014;25(6):846-859.

26. Wanderley CW, et al. Paclitaxel reduces tumor growth by reprogramming tumor-associated macrophages to an M1 profile in a TLR4-dependent manner. Cancer Res. 2018;78(20):5891-5900.

27. Mestas J, Ley K. Monocyte-endothelial cell interactions in the development of atherosclerosis. Trends Cardiovasc Med. 2008;18(6):228-232.

28. Huo Y, Ley K. Adhesion molecules and atherogenesis. Acta Physiol Scand. 2001;173(1):35-43.

29. Shattil SJ, Ginsberg MH. Perspectives series: cell adhesion in vascular biology. Integrin signaling in vascular biology. J Clin Invest. 1997;100(1):1-5.

30. Elices M, et al. VCAM1 on activated endothelium interacts with the leukocyte integrin VLA4 at a site distinct from the VLA4/fibronectin binding site. Cell. 1990;60(4):577-584.

31. Osborn L, et al. Direct expression cloning of vascular cell adhesion molecule 1 , a cytokine-induced endothelial protein that binds to lymphocytes. Cell. 1989;59(6):1203-1211.

32. Newman G, Pudney D. A survey of current practice in the management of recurrent malignant ascites among oncologists and palliative-care physicians in the UK. Clin Oncol (R Coll Radiol). 2006;18(2):154.

33. Becker G, et al. Malignant ascites: systematic review and guideline for treatment. Eur J Cancer. 2006;42(5):589-597.

34. Ostrowski MJ. An assessment of the long-term results of controlling the reaccumulation of malignant effusions using intracavity bleomycin. Cancer. 1986;57(4):721-727.

35. Maiche AG. Management of peritoneal effusions with intracavitary mitoxantrone or bleomycin. Anticancer Drugs. 1994;5(3):305-308.

36. Link KH, et al. Intraperitoneal chemotherapy with mitoxantrone in malignant ascites. Surg Oncol Clin N Am. 2003;12(3):865-872.

37. Schilsky RL, et al. Phase I clinical and pharmacologic study of intraperitoneal cisplatin and fluorouracil in patients with advanced intraabdominal cancer. JClin Oncol. 1990;8(12):2054-2061.

38. Sennino B, McDonald DM. Controlling escape from angiogenesis inhibitors. Nat Rev Cancer. 2012;12(10):699-709. 